\title{
COUNTING ORDERED PATTERNS IN WORDS GENERATED BY MORPHISMS
}

\author{
Sergey Kitaev \\ Department of Mathematics, Reykjavik University, Kringlan 1, 103 Reykjavik, Iceland \\ sergey@ru. is \\ Toufik Mansour \\ Department of Mathematics, Haifa University, 31905 Haifa, Israel \\ toufik@math.haifa.ac.il \\ Patrice Séébold \\ Département Mathématiques Informatique et Applications, Université Paul Valéry, Route de Mende, 34199 \\ Montpellier Cédex 5, France \\ and \\ LIRMM, Univ. Montpellier 2, CNRS, 161 rue Ada, 34392 Montpellier, France \\ Patrice.Seebold@lirmm.fr
}

Received: 9/21/07, Accepted: 1/18/08, Published: 1/29/08

\begin{abstract}
We start a general study of counting the number of occurrences of ordered patterns in words generated by morphisms. We consider certain patterns with gaps (classical patterns) and those with no gaps (consecutive patterns). Occurrences of the patterns are known, in the literature, as rises, descents, (non-)inversions, squares and $p$-repetitions. We give recurrence formulas in the general case, then deducing exact formulas for particular families of morphisms. Many (classical or new) examples are given illustrating the techniques and showing their interest.
\end{abstract}

\section{Introduction}

Different notions of pattern can be encountered in several domains of combinatorics. In algebraic combinatorics, an occurrence of a pattern $p$ in a permutation $\pi$ is a subsequence of $\pi$ (of the same length as the length of $p$ ) whose elements are in the same relative order as those in $p$. For example, the permutation $\pi=536241$ contains an occurrence of the pattern 
$p=2431$ : indeed the elements of the subsequence 3641 of $\pi$ are in the same relative order as those in $p$. Examples of results concern permutations avoiding a pattern of length 3 in the symmetric group $S_{n}$ (see $\left.[19,30]\right)$.

Motivated by the study of Mahonian statistics, Babson and Steingrímsson introduced a generalization where two adjacent elements of the pattern must also be adjacent in the permutation [4]. In [11], this generalization provides interesting results related to set partitions, Dyck paths, Motzkin paths, or involutions.

In combinatorics on words, an occurrence of a pattern $p$ in a word $u$ is a factor of $u$ having the same shape as $p$, i.e., for which there exists a nonerasing morphism transforming $p$ in this factor. For example the word $u=a b a a b a a a b a b$ contains an occurrence of the pattern $p=\alpha \alpha \beta \alpha \alpha \beta$ : indeed the morphism $f(\alpha)=a, f(\beta)=b a$ transforms the pattern $p$ in aabaaaba which is a factor of $u$. The main question is to determine whether or not a given pattern is unavoidable, that is if it is possible to construct an infinite word containing no occurrence of the pattern. The interested reader should refer to Chapter 3 of [22].

In [7-10] the authors realized a "mixing" of these two notions. They consider ordered alphabets. Here, an occurrence of a pattern in a word is a factor or a subsequence having the same shape, and in which the relative order of the letters is the same as in the pattern. For example, on the alphabet $\{a, b\}$ with $a<b$, the word $u=a b a a a b a b$ contains an occurrence of the pattern 2111 (the factor baaa) but not of the pattern 1222 (abbb is not a factor of $u$ ). However, the word $u$ contains an occurrence of the pattern with gaps $1 \# 2 \# 2 \# 2$ because $a b b b$ is a subsequence of $u$ (here \# means that the letters corresponding to 1 and 2 may be not consecutive). To avoid confusion with previous notions we call these patterns ordered patterns (with gaps if there is at least one \#, with no gaps if there is no \#).

In [18] we computed the number of occurrences of a lot of ordered patterns in the Peano words (words corresponding to finite approximations of the Peano space filling curve). An interesting property of these words is that they are generated by a tag-system, i.e., by applying two morphisms. A motivation for this choice is the interest in studying classes of words defined by iterative schemes, in particular with morphisms that are a fundamental tool of formal languages [2, 22, 27].

In the present paper we start a general study of counting the number of occurrences of ordered patterns in words generated by morphisms. After some preliminaries (Section 2), we give in Section 3 some general results (recurrence formulas) on counting elementary ordered patterns with gaps ((non-)inversions and p-repetitions) in words generated by morphisms, and applications to two well known binary words. Section 4 is dedicated to more precise results (exact formulas) in the case of a particular family of morphisms, and in Section 5 we give many examples of morphisms belonging to this family. Section 6 is dedicated to counting elementary ordered patterns with no gaps (rises, descents, and squares) in words generated by morphisms and giving some examples. 


\section{Preliminaries}

\subsection{Definitions and Notations}

The terminology and notations are mainly those of Lothaire [22].

Let $A$ be a finite set called the alphabet and $A^{*}$ the free monoid generated by $A$. The elements of $A$ are called letters and those of $A^{*}$ are called words. The empty word $\varepsilon$ is the neutral element of $A^{*}$ for the concatenation of words (the concatenation of two words $u$ and $v$ is the word $u v$ ), and we denote by $A^{+}$the semigroup $A^{*} \backslash\{\varepsilon\}$.

The length of a word $u$, denoted by $|u|$, is the number of occurrences of letters in $u$. In particular $|\varepsilon|=0$. If $n$ is a nonnegative integer, $u^{n}$ is the word obtained by concatenating $n$ occurrences of the word $u$. Of course, $\left|u^{n}\right|=n \cdot|u|$. The cases $n=2$, and $n=3$ deserve a particular attention in what follows. A word $u^{2}$ (resp. $u^{3}$ ), with $u \neq \varepsilon$, is called a square (resp. a cube).

A word $w$ is called a factor (resp. a prefix) of $u$ if there exist words $x, y$ such that $u=x w y$ (resp. $u=w y$ ). The factor (resp. the prefix) is proper if $x y \neq \varepsilon$ (resp. $y \neq \varepsilon$ ). The number of distinct occurrences of $w$ in $u$ is denoted by $|u|_{w}$. A word $u$ is a subsequence of the word $v$ if there exist words $u_{1}, \ldots, u_{n}, v_{1}, \ldots, v_{n}, v_{n+1}$ such that $u=u_{1} \cdots u_{n}$ and $v=v_{1} u_{1} v_{2} u_{2} \cdots v_{n} u_{n} v_{n+1}$.

An infinite word (or sequence) over $A$ is an application $\mathbf{a}: \mathbb{N} \rightarrow A$. It is written $\mathbf{a}=$ $a_{0} a_{1} \cdots a_{i} \cdots, i \in \mathbb{N}, a_{i} \in A$.

The notion of factor is extended to infinite words as follows: a (finite) word $u$ is a factor (resp. prefix) of an infinite word a over $A$ if there exist $n \in \mathbb{N}$ (resp. $n=0$ ) and $m \in \mathbb{N}$ $(m=|u|)$ such that $u=a_{n} \cdots a_{n+m-1}$ (by convention $a_{n} \cdots a_{n-1}=\varepsilon$ ).

In what follows, we will consider morphisms on $A$. Let $B$ be an alphabet (often, $B=A$ ).

A morphism on $A$ is an application $f: A^{*} \rightarrow B^{*}$ such that $f(u v)=f(u) f(v)$ for all $u, v \in A^{*}$. It is uniquely determined by its value on the alphabet $A$. A morphism $f$ on $A$ is a literal morphism if $|f(a)|=1$ for all $a \in A$.

For $A=B$, let $n$ be a non-negative integer. The incidence matrix of $f^{n}$ is the $k \times k$ matrix

$$
M\left(f^{n}\right)=\left(m_{n, i, j}\right)_{1 \leq i, j \leq k}
$$

where $m_{n, i, j}$ is the number of occurrences of the letter $a_{i}$ in the word $f^{n}\left(a_{j}\right)$, i.e., $m_{n, i, j}=$ $\left|f^{n}\left(a_{j}\right)\right|_{a_{i}}$. For details on the incidence matrix of a morphism see, e.g., [2], chapter 8, in which is given the following.

Property 1 For every $n \in \mathbb{N}, M(f)^{n}=M\left(f^{n}\right)$. 
A morphism is nonerasing if $f(a) \neq \varepsilon$ for all $a \in A$. Such a morphism is prolongable on $x_{0}, x_{0} \in A^{+}$, if there exists $u \in A^{+}$such that $f\left(x_{0}\right)=x_{0} u$. In this case, for all $n \in \mathbb{N}$ the word $f^{n}\left(x_{0}\right)$ is a proper prefix of the word $f^{n+1}\left(x_{0}\right)$ and this defines a unique infinite word

$$
\mathbf{x}=x_{0} u f(u) f^{2}(u) \cdots f^{n}(u) \cdots
$$

which is the limit of the sequence $\left(f^{n}\left(x_{0}\right)\right)_{n \geq 0}$. We write $\mathbf{x}=f^{\omega}\left(x_{0}\right)$ and say that $\mathbf{x}$ is generated by $f$.

A (finite or infinite) word $u$ over $A$ is square-free (resp. cube-free) if none of its factors is a square (resp. a cube). A morphism $f$ on $A$ is square-free if the word $f(u)$ is square-free whenever $u$ is a square-free word. The morphism $f$ is weakly square-free if $f$ generates a square-free infinite word.

A tag-system is a quintuple $T=(A, u, f, g, B)$ where $A$ and $B$ are alphabets, $u \in A^{+}, f$ is a nonerasing morphism on $A$, prolongable on $u$, and $g$ is a morphism from $A$ onto $B$. An infinite word $\mathbf{y}$ is generated by $G$ if $\mathbf{y}=g\left(\left(f^{k}\right)^{\omega}(u)\right)$ for some $k \in \mathbb{N}$.

Remark that what we call here a tag-system is sometimes called a HDOL-system. The terminology of tag-system comes from the fundamental study of Cobham [12]. Chapter 5 of [26] is dedicated to a deep study of D0L-systems.

\subsection{Ordered Patterns}

Let $A$ be a totally ordered alphabet and let $\aleph$ be the ordered alphabet whose letters are the first $n$ positive integers in the usual order (thus $\aleph=\{1,2, \ldots, n\}$ ). An ordered pattern is any word $^{1}$ over $\aleph \cup\{\#\}, \# \notin \aleph$, without two consecutive \#. If a pattern contains at least one \#, not at the very beginning or at the very end, it is an ordered pattern with gaps; otherwise it is an ordered pattern with no gaps ${ }^{2}$. Moreover, in this paper the ordered patterns $u$, \#u, $u \#$, and $\# u \#$ are considered to be the same. In particular, if $x$ is a word over $\aleph$, we will write $(x \#)^{\ell}$ or $(\# x)^{\ell}$ to represent the ordered pattern $x \# x \# \cdots \# x$ containing $l$ occurrences of the word $x$.

A word $v$ over $A$ contains an occurrence of the ordered pattern $u=u_{1} \# u_{2} \# \cdots \# u_{n}$, where $u_{i} \in \aleph^{+}$and $n \geq 1$, (or, equivalently the ordered pattern $u$ occurs in $v$ ) if $v=$ $w_{0} v_{1} w_{1} v_{2} w_{2} \cdots w_{n-1} v_{n} w_{n}$ and there exists a literal morphism $f: \aleph^{*} \rightarrow A^{*}$ such that $f\left(u_{i}\right)=$ $v_{i}, 1 \leq i \leq n$, and if $x, y \in \aleph, x<y \Rightarrow f(x)<f(y)$. Thus the word $v$ contains an occurrence

\footnotetext{
${ }^{1}$ In algebraic combinatorics when defining a pattern it is claimed that each letter from the interval $[k]$ must occur at least once. This requirement is not useful here, what is important is the relative value of each letter because this gives the order. However it will be often implicit that these letters (which are only formal representations of the pattern) are taken in the order from 1.

${ }^{2}$ Our choice here is to use terminology of combinatorics on words. For example, our notion of pattern with no gaps is often referred to as pattern without internal dashes or consecutive pattern in the literature about algebraic combinatorics (see, e.g., [17]). However this terminology does not seem to be solid since Burstein and Mansour used subword pattern without hyphens [10], and segmented pattern is also encountered.
} 
of the ordered pattern $u$ if $v$ contains a subsequence $v^{\prime}$ which is equal to $f\left(u^{\prime}\right)$ where $u^{\prime}$ is obtained from $u$ by deleting all the occurrences of \#, with the additional condition that two adjacent (not separated by $\#$ ) letters in $u$ must be adjacent in $v$. The number of different occurrences of $u$ as an ordered pattern in $v$ is denoted by $|v|_{u}$ (this is consistent with the classical notation because if $u$ is a single letter then $|v|_{u}$ is just the number of occurrences of the letter $u$ in the word $v$ ).

Example. Let $A=\{a, b, c, d, e, f\}$ with $a<b<c<d<e<f$. The word $v=e a f d b c$ contains one occurrence of the ordered pattern $2 \# 31$, namely the subsequence efd $\left(|e a f d b c|_{2 \# 31}=\right.$ 1). In $v$, the ordered pattern $2 \# 3 \# 1$ occurs in three occurrences: efd, ef $b$, and efc $\left(|e a f d b c|_{2 \# 3 \# 1}=3\right)$; the ordered pattern 231 does not occur in $v\left(|e a f d b c|_{231}=0\right)$.

\section{Ordered Patterns with Gaps and Morphisms}

Let $k$ be an integer $(k \geq 2)$ and $A$ the $k$-letter ordered alphabet $A=\left\{a_{1}<a_{2}<\cdots<a_{k}\right\}$. Let $f$ be any morphism on $A$ : for $1 \leq i \leq k, f\left(a_{i}\right)=a_{i_{1}} \ldots a_{i_{p_{i}}}$ with $p_{i} \geq 0\left(p_{i}=0\right.$ if and only if $\left.f\left(a_{i}\right)=\varepsilon\right)$.

\subsection{Inversions, Non-inversions, and Repetitions with Gaps of $f^{n}$}

In what follows we are interested in some particular forms of ordered patterns. In accordance with permutations theory, an inversion (resp. non-inversion) is an occurrence of the ordered pattern $2 \# 1$ (resp. 1\#2). Repetitions with gaps of one letter are occurrences of the ordered patterns $(1 \#)^{p}, p \geq 1$.

\subsubsection{Inversions and Non-inversions}

Let $n$ be a non-negative integer. The vector ${ }^{3}$ of non-inversions (denoted by $R G$ for "rises with gaps") of $f^{n}$ is the $k$ vector whose $i$-th entry is the number of occurrences of the ordered pattern $1 \# 2$ in the word $f^{n}\left(a_{i}\right)$, i.e.,

$$
R G\left(f^{n}\right)=\left(\left|f^{n}\left(a_{i}\right)\right|_{1 \# 2}\right)_{1 \leq i \leq k} .
$$

The vector of inversions (denoted by $D G$ for "descents with gaps") of $f^{n}$ is the $k$ vector whose $i$-th entry is the number of occurrences of the ordered pattern $2 \# 1$ in the word $f^{n}\left(a_{i}\right)$,

\footnotetext{
${ }^{3}$ The computation of the number of ordered patterns in words is related to so-called Parikh matrices. For example the number of occurrences of the ordered pattern $1 \# 2$ in a word $w$ over a three-letter alphabet is equal to $M_{1,3}+M_{2,4}$ where $M$ is the Parikh matrix of $w$ (for more information on Parikh matrices see, e.g., Mateescu et al. [23] or Fossé and Richomme [14]). However Parikh matrices are not well adapted to compute the number of most of the ordered patterns (for example in the case of ordered patterns as $1 \# 2 \# 1$ or 1\#23) which explains that here we use another more convenient representation with vectors.
} 
i.e.,

$$
D G\left(f^{n}\right)=\left(\left|f^{n}\left(a_{i}\right)\right|_{2 \# 1}\right)_{1 \leq i \leq k}
$$

Our goal is to obtain recurrence formulas giving the entries of $R G\left(f^{n+1}\right)$ and $D G\left(f^{n+1}\right)$. Since $f^{n+1}=f^{n} \circ f=f \circ f^{n}$, we have two different ways to compute $R G\left(f^{n+1}\right)$ and $D G\left(f^{n+1}\right)$.

Let $\ell$ be an integer, $1 \leq \ell \leq k$. Either $\left|f^{n+1}\left(a_{\ell}\right)\right|_{1 \# 2}$ (resp. $\left.\left|f^{n+1}\left(a_{\ell}\right)\right|_{2 \# 1}\right)$ will be obtained from the value of $f\left(a_{\ell}\right)$ and the entries of $R G\left(f^{n}\right)$ (resp. $D G\left(f^{n}\right)$ ) (see 1. below), or it will be computed from the values of $R G(f)$ (resp. $D G(f))$ and $f^{n}\left(a_{\ell}\right)$ (see 2 . below).

1. From $f^{n+1}=f^{n} \circ f$.

Since $f\left(a_{\ell}\right)=a_{\ell_{1}} \ldots a_{\ell_{p_{\ell}}}$, the number of occurrences of the ordered pattern $1 \# 2$ in $f^{n+1}\left(a_{\ell}\right)=f^{n}\left(f\left(a_{\ell}\right)\right)=f^{n}\left(a_{\ell_{1}} \ldots a_{\ell_{p_{\ell}}}\right)$ is obtained by adding two values:

- the number of occurrences of the ordered pattern $1 \# 2$ in each $f^{n}\left(a_{\ell_{i}}\right), 1 \leq i \leq p_{\ell}$. Since the $\ell$-th column of the incidence matrix of $f$ indicates which letters appear in $f\left(a_{\ell}\right)$ (and how many times), this number is obtained by multiplying the vector $R G\left(f^{n}\right)$ by the $\ell$-th column of $M(f)$, i.e., it is equal to $\sum_{t=1}^{k}\left|f^{n}\left(a_{t}\right)\right|_{1 \# 2} \cdot m_{1, t, \ell}$,

- the number of occurrences of the ordered pattern $1 \# 2$ in each of the $f^{n}\left(a_{\ell_{i}} a_{\ell_{j}}\right)$, $1 \leq i<j \leq p_{\ell}$, where the letter corresponding to 1 is in $f^{n}\left(a_{\ell_{i}}\right)$ and the letter corresponding to 2 is in $f^{n}\left(a_{\ell_{j}}\right)$. In what follows we will call such an occurrence of $1 \# 2$ in $f^{n}\left(a_{\ell_{i}} a_{\ell_{j}}\right)$ an external occurrence of the ordered pattern $1 \# 2$ in $f^{n}\left(a_{\ell_{i}} a_{\ell_{j}}\right)$, and denote it $\left|f^{n}\left(a_{\ell_{i}} a_{\ell_{j}}\right)\right|_{1 \# 2}^{e x t}$.

The value of $\left|f^{n}\left(a_{\ell_{i}} a_{\ell_{j}}\right)\right|_{1 \# 2}^{e x t}$ is obtained by adding, for all the integers $r, 1 \leq$ $r \leq k-1$, the product of the number of occurrences of the letter $a_{r}$ in $f^{n}\left(a_{\ell_{i}}\right)$ $\left(\left|f^{n}\left(a_{\ell_{i}}\right)\right|_{a_{r}}\right)$ by the number of occurrences of all the letters of $f^{n}\left(a_{\ell_{j}}\right)$ greater than $a_{r}\left(\left|f^{n}\left(a_{\ell_{j}}\right)\right|_{a_{s}}, r+1 \leq s \leq k\right)$. This gives $\sum_{r=1}^{k-1}\left(m_{n, r, \ell_{i}} \cdot \sum_{s=r+1}^{k} m_{n, s, \ell_{j}}\right)$.

The number of external occurrences of $1 \# 2$ in all the $f^{n}\left(a_{\ell_{i}} a_{\ell_{j}}\right), 1 \leq i<j \leq p_{\ell}$, is thus given by $\sum_{1 \leq i<j \leq p_{\ell}}\left|f^{n}\left(a_{\ell_{i}} a_{\ell_{j}}\right)\right|_{1 \# 2}^{e x t}=\sum_{1 \leq i<j \leq p_{\ell}}\left(\sum_{r=1}^{k-1}\left(m_{n, r, \ell_{i}} \cdot \sum_{s=r+1}^{k} m_{n, s, \ell_{j}}\right)\right)$.

2. From $f^{n+1}=f \circ f^{n}$.

Let $q_{\ell}=\left|f^{n}\left(a_{\ell}\right)\right|: f^{n+1}\left(a_{\ell}\right)=f\left(f^{n}\left(a_{\ell}\right)\right)=f\left(a_{\ell_{1}^{\prime}} \ldots a_{\ell_{q_{\ell}}}\right)$. Here the number of occurrences of the ordered pattern $1 \# 2$ in $f^{n+1}\left(a_{\ell}\right)$ is obtained by adding

- the number of occurrences of the ordered pattern $1 \# 2$ in each $f\left(a_{\ell_{i}^{\prime}}\right), 1 \leq i \leq q_{\ell}$. Since the $\ell$-th column of the incidence matrix of $f^{n}$ indicates which letters appear in $f^{n}\left(a_{\ell}\right)$ (and how many times), this number is obtained by multiplying the vector $R G(f)$ by the $\ell$-th column of $M\left(f^{n}\right)$, i.e., it is equal to $\sum_{t=1}^{k}\left|f\left(a_{t}\right)\right|_{1 \# 2} \cdot m_{n, t, \ell}$,

- the number of external occurrences of the ordered pattern $1 \# 2$ in each of the $f\left(a_{\ell_{i}^{\prime}} a_{\ell_{j}^{\prime}}\right), 1 \leq i<j \leq q_{\ell}$. This number is obtained by adding, for all the integers $r, 1 \leq r \leq k-1$, the product of the number of occurrences of the letter $a_{r}$ in 
$f\left(a_{\ell_{i}^{\prime}}\right)\left(\left|f\left(a_{\ell_{i}^{\prime}}\right)\right|_{a_{r}}\right)$ by the number of occurrences of all the letters of $f\left(a_{\ell_{j}^{\prime}}\right)$ greater than $a_{r}\left(\left|f\left(a_{\ell_{j}^{\prime}}\right)\right|_{a_{s}}, r+1 \leq s \leq k\right)$. This gives $\sum_{r=1}^{k-1}\left(m_{1, r, \ell_{i}^{\prime}} \cdot \sum_{s=r+1}^{k} m_{1, s, \ell_{j}^{\prime}}\right)$.

The number of external occurrences of $1 \# 2$ in all the $f\left(a_{\ell_{i}^{\prime}} a_{\ell_{j}^{\prime}}\right), 1 \leq i<j \leq q_{\ell}$, is thus given by $\sum_{1 \leq i<j \leq q_{\ell}}\left|f\left(a_{\ell_{i}^{\prime}} a_{\ell_{j}^{\prime}}\right)\right|_{1 \# 2}^{e x t}=\sum_{1 \leq i<j \leq q \ell}\left(\sum_{r=1}^{k-1}\left(m_{1, r, \ell_{i}^{\prime}} \cdot \sum_{s=r+1}^{k} m_{1, s, \ell_{j}^{\prime}}\right)\right)$.

The same reasoning applies for calculating the entries of $D G\left(f^{n+1}\right)$, replacing $1 \# 2$ by $2 \# 1$ and "greater" by "smaller".

Thus, we have the following.

Proposition 1 For each letter $a_{\ell} \in A$, let $p_{\ell}$ and $q_{\ell}$ be such that $f\left(a_{\ell}\right)=a_{\ell_{1}} \ldots a_{\ell_{p_{\ell}}}$ and $f^{n}\left(a_{\ell}\right)=a_{\ell_{1}^{\prime}} \ldots a_{\ell_{q_{\ell}}^{\prime}}$. Then, for all $n \in \mathbb{N}$,

$$
\begin{aligned}
\left|f^{n+1}\left(a_{\ell}\right)\right|_{1 \# 2} & =\sum_{1 \leq i<j \leq p_{\ell}}\left(\sum_{r=1}^{k-1}\left(m_{n, r, \ell_{i}} \cdot \sum_{s=r+1}^{k} m_{n, s, \ell_{j}}\right)\right)+\sum_{t=1}^{k}\left|f^{n}\left(a_{t}\right)\right|_{1 \# 2} \cdot m_{1, t, \ell}, \\
& =\sum_{1 \leq i<j \leq q \ell}\left(\sum_{r=1}^{k-1}\left(m_{1, r, \ell_{i}^{\prime}} \cdot \sum_{s=r+1}^{k} m_{1, s, \ell_{j}^{\prime}}\right)\right)+\sum_{t=1}^{k}\left|f\left(a_{t}\right)\right|_{1 \# 2} \cdot m_{n, t, \ell}, \\
\left|f^{n+1}\left(a_{\ell}\right)\right|_{2 \# 1} & =\sum_{1 \leq i<j \leq p_{\ell}}\left(\sum_{r=2}^{k}\left(m_{n, r, \ell_{i}} \cdot \sum_{s=1}^{r-1} m_{n, s, \ell_{j}}\right)\right)+\sum_{t=1}^{k}\left|f^{n}\left(a_{t}\right)\right|_{2 \# 1} \cdot m_{1, t, \ell} \\
& =\sum_{1 \leq i<j \leq q_{\ell}}\left(\sum_{r=2}^{k}\left(m_{1, r, \ell_{i}^{\prime}} \cdot \sum_{s=1}^{r-1} m_{1, s, \ell_{j}^{\prime}}\right)\right)+\sum_{t=1}^{k}\left|f\left(a_{t}\right)\right|_{2 \# 1} \cdot m_{n, t, \ell} .
\end{aligned}
$$

Of course, the analysis we have done above could be realized to compute more complex ordered patterns with gaps, such as $1 \# 23,1 \# 2 \# 3, \cdots$ The only difficulty is to adapt the computation of external inversions and non-inversions.

\subsubsection{Repetitions of One Letter}

Let $n$ be a non-negative integer and $p$ a positive integer. The vector of $p$-repetitions with gaps of one letter of $f^{n}$ is the $k$ vector whose $i$-th entry is the number of occurrences of the ordered pattern $(1 \#)^{p}$ in the word $f^{n}\left(a_{i}\right)$, i.e.,

$$
R_{p} G\left(f^{n}\right)=\left(\left|f^{n}\left(a_{i}\right)\right|_{(1 \#)^{p}}\right)_{1 \leq i \leq k} .
$$

The following is obvious.

Proposition 2 For each letter $a_{\ell} \in A$ and for all $n \in \mathbb{N}$,

$$
\left|f^{n}\left(a_{\ell}\right)\right|_{(1 \#)^{p}}=\sum_{t=1}^{k}\left(\begin{array}{c}
m_{n, t, \ell} \\
p
\end{array}\right) .
$$




\subsection{Some Examples in the Binary Case}

Since equations (1) to (5) are recurrence formulas they are not always suitable to produce exact formulas giving the entries of $R G\left(f^{n}\right), D G\left(f^{n}\right)$, and $R_{p} G\left(f^{n}\right)$. However, in some particular cases we obtained such exact formulas. This is in particular the case for the following two classical morphisms on the two-letter ordered alphabet $\left\{a_{1}<a_{2}\right\}$.

\subsubsection{The Thue-Morse Morphism}

The Thue-Morse morphism $\mu$ was introduced in 1912 by Thue [31], although it was hinted at sixty years earlier by Prouhet [25]. It was discovered independently in 1921 by Morse [24]. This morphism is defined by $\mu\left(a_{1}\right)=a_{1} a_{2}, \mu\left(a_{2}\right)=a_{2} a_{1}$. It generates the famous Thue-Morse sequence $\mathbf{t}=\mu^{\omega}\left(a_{1}\right)$ which has been widely studied (see, e.g., Lothaire [21], or Allouche and Shallit [2], and references therein).

For every positive integers $n$, the incidence matrix of $\mu^{n}$ is $M\left(\mu^{n}\right)=\left[\begin{array}{ll}2^{n-1} & 2^{n-1} \\ 2^{n-1} & 2^{n-1}\end{array}\right]$. Thus, from equations (1), (3), and (5) we obtain

$$
\begin{aligned}
\left|\mu^{n+1}\left(a_{1}\right)\right|_{1 \# 2} & =\left|\mu^{n+1}\left(a_{2}\right)\right|_{1 \# 2}=2^{2(n-1)}+\left|\mu^{n}\left(a_{1}\right)\right|_{1 \# 2}+\left|\mu^{n}\left(a_{2}\right)\right|_{1 \# 2}, \\
\left|\mu^{n+1}\left(a_{1}\right)\right|_{2 \# 1} & =\left|\mu^{n+1}\left(a_{2}\right)\right|_{2 \# 1}=2^{2(n-1)}+\left|\mu^{n}\left(a_{1}\right)\right|_{2 \# 1}+\left|\mu^{n}\left(a_{2}\right)\right|_{2 \# 1}, \\
\left|\mu^{n}\left(a_{1}\right)\right|_{(1 \#)^{p}} & =\left|\mu^{n}\left(a_{2}\right)\right|_{(1 \#)^{p}}=2 \cdot\left(\begin{array}{c}
2^{n-1} \\
p
\end{array}\right) .
\end{aligned}
$$

Since $R G(\mu)=\left[\begin{array}{ll}1 & 0\end{array}\right]$ and $D G(\mu)=\left[\begin{array}{ll}0 & 1\end{array}\right]$, we obtain from Proposition 1 the following trivial result.

Corollary 1 For any integer $n \geq 2$,

$$
R G\left(\mu^{n}\right)=D G\left(\mu^{n}\right)=\left[\begin{array}{ll}
2^{2 n-3} & 2^{2 n-3}
\end{array}\right] \text { and } R_{p} G\left(\mu^{n}\right)=\left[\begin{array}{cc}
2 \cdot\left(\begin{array}{c}
2^{n-1} \\
p
\end{array}\right) & 2 \cdot\left(\begin{array}{c}
2^{n-1} \\
p
\end{array}\right)
\end{array}\right] .
$$

\subsubsection{The Fibonacci Morphism}

The Fibonacci morphism $\varphi$ is defined by $\varphi\left(a_{1}\right)=a_{1} a_{2}, \varphi\left(a_{2}\right)=a_{1}$. It generates the well known Fibonacci sequence $\mathbf{f}=\varphi^{\omega}\left(a_{1}\right)$ which has numerous remarkable properties and is the prototype of a Sturmian word (see, e.g., Chapter 2 of [22]).

Let $\left(F_{n}\right)_{n \geq-1}$ be the sequence of Fibonacci numbers: $F_{-1}=0, F_{0}=1, F_{n}=F_{n-1}+F_{n-2}$ for $n \geq 1$. The following property of Fibonacci numbers will be useful below.

Property 2 For every positive integer $n$,

$$
F_{n} F_{n-2}=F_{n-1}^{2}+\left\{\begin{aligned}
1 & \text { if } n \text { is even, } \\
-1 & \text { if } n \text { is odd. }
\end{aligned}\right.
$$


An easy computation gives, for every positive integer $n$, the incidence matrix of $\varphi^{n}$ :

$$
M\left(\varphi^{n}\right)=\left[\begin{array}{ll}
F_{n} & F_{n-1} \\
F_{n-1} & F_{n-2}
\end{array}\right] .
$$

The vector of non-inversions of $\varphi$ is $R G(\varphi)=\left[\begin{array}{ll}1 & 0\end{array}\right]$. Moreover, from equation (1), we obtain for $n \geq 1$

$$
\begin{aligned}
\left|\varphi^{n+1}\left(a_{1}\right)\right|_{1 \# 2} & =m_{n, 1,1} \cdot m_{n, 2,2}+\left|\varphi^{n}\left(a_{1}\right)\right|_{1 \# 2}+\left|\varphi^{n}\left(a_{2}\right)\right|_{1 \# 2} \\
& =F_{n} F_{n-2}+\left|\varphi^{n}\left(a_{1}\right)\right|_{1 \# 2}+\left|\varphi^{n}\left(a_{2}\right)\right|_{1 \# 2} \\
& =F_{n-1}^{2}+\left|\varphi^{n}\left(a_{1}\right)\right|_{1 \# 2}+\left|\varphi^{n}\left(a_{2}\right)\right|_{1 \# 2}+\left\{\begin{aligned}
1 & \text { if } n \text { is even, } \\
-1 & \text { if } n \text { is odd }
\end{aligned}\right. \text { (see Property 2). }
\end{aligned}
$$

The vector of inversions of $\varphi$ is $D G(\varphi)=\left[\begin{array}{ll}0 & 0\end{array}\right]$. Moreover, from equation (3), we obtain for $n \geq 1$

$$
\begin{aligned}
\left|\varphi^{n+1}\left(a_{1}\right)\right|_{2 \# 1} & =m_{n, 2,1} \cdot m_{n, 1,2}+\left|\varphi^{n}\left(a_{1}\right)\right|_{2 \# 1}+\left|\varphi^{n}\left(a_{2}\right)\right|_{2 \# 1} \\
& =F_{n-1}^{2}+\left|\varphi^{n}\left(a_{1}\right)\right|_{2 \# 1}+\left|\varphi^{n}\left(a_{2}\right)\right|_{2 \# 1} .
\end{aligned}
$$

Now, $\left|\varphi^{n+1}\left(a_{2}\right)\right|_{1 \# 2}=\left|\varphi^{n}\left(a_{1}\right)\right|_{1 \# 2}$ and $\left|\varphi^{n+1}\left(a_{2}\right)\right|_{2 \# 1}=\left|\varphi^{n}\left(a_{1}\right)\right|_{2 \# 1}$ because $\varphi\left(a_{2}\right)=$ $a_{1}$. From this we obtain direct formulas to compute, for every $n \geq 0,\left|\varphi^{n+2}\left(a_{1}\right)\right|_{1 \# 2}$ and $\left|\varphi^{n+2}\left(a_{1}\right)\right|_{2 \# 1}$ from the sequence of Fibonacci numbers.

Corollary 2 For every integer $n \geq 0$,

$$
\begin{aligned}
\left|\varphi^{n+2}\left(a_{1}\right)\right|_{2 \# 1} & =\sum_{p=0}^{n} F_{p} F_{n-p}^{2}, \\
\left|\varphi^{n+2}\left(a_{1}\right)\right|_{1 \# 2} & =\left|\varphi^{n+2}\left(a_{1}\right)\right|_{2 \# 1}+F_{n}+\left\{\begin{aligned}
1 & \text { if } n \text { is odd, } \\
-1 & \text { if } n \text { is even. }
\end{aligned}\right.
\end{aligned}
$$

Proof. Since $F_{0}=1$ and $\varphi^{2}\left(a_{1}\right)=a_{1} a_{2} a_{1}$, the result is obviously true if $n=0$. Also, since $F_{0}=1, F_{1}=1$, and $\varphi^{3}\left(a_{1}\right)=a_{1} a_{2} a_{1} a_{1} a_{2}$, the result is true for $n=1$. Now suppose $n \geq 2$ and the assertions are true for all $m<n$. We prove they are true for $n$.

- We first compute $\left|\varphi^{n+2}\left(a_{1}\right)\right|_{2 \# 1}$.

$$
\begin{aligned}
\left|\varphi^{n+2}\left(a_{1}\right)\right|_{2 \# 1}=F_{n}^{2} & +\left|\varphi^{n+1}\left(a_{1}\right)\right|_{2 \# 1}+\left|\varphi^{n}\left(a_{1}\right)\right|_{2 \# 1} \\
=F_{n}^{2} & +\sum_{p=0}^{n-1} F_{p} F_{n-1-p}^{2}+\sum_{p=0}^{n-2} F_{p} F_{n-2-p}^{2} . \\
\text { But } \sum_{p=0}^{n-2} F_{p} F_{n-2-p}^{2} & =\sum_{p=1}^{n-1} F_{p-1} F_{n-2-(p-1)}^{2} \\
& =\sum_{p=1}^{n-1} F_{p-1} F_{n-1-p}^{2} . \\
\text { Thus }\left|\varphi^{n+2}\left(a_{1}\right)\right|_{2 \# 1} & =F_{n}^{2}+F_{0} F_{n-1}^{2}+\sum_{p=1}^{n-1}\left(F_{p}+F_{p-1}\right) F_{n-1-p}^{2} \\
& =F_{n}^{2}+F_{n-1}^{2}+\sum_{p=1}^{n-1} F_{p+1} F_{n-(p+1)}^{2} \\
& =F_{n}^{2}+F_{n-1}^{2}+\sum_{p=2}^{n} F_{p} F_{n-p}^{2} \\
& =\sum_{p=0}^{n} F_{p} F_{n-p}^{2}\left(\text { because } F_{0}=F_{1}=1\right) .
\end{aligned}
$$


- For $\left|\varphi^{n+2}\left(a_{1}\right)\right|_{1 \# 2}$, we remark that if $n$ is even then $n-2$ is even, and $n-1, n+1$ are odd. And if $n$ is odd then $n-2$ is odd, and $n-1, n+1$ are even. Consequently,

$$
\begin{aligned}
& \left|\varphi^{n+2}\left(a_{1}\right)\right|_{1 \# 2}=F_{n}^{2}+\left|\varphi^{n+1}\left(a_{1}\right)\right|_{1 \# 2}+\left|\varphi^{n}\left(a_{2}\right)\right|_{1 \# 2}+\left\{\begin{aligned}
1 & \text { if } n+1 \text { is even }(n \text { odd }), \\
-1 & \text { if } n+1 \text { is odd }(n \text { even })
\end{aligned}\right. \\
& =F_{n}^{2}+\sum_{p=0}^{n-1} F_{p} F_{n-1-p}^{2}+F_{n-1}+1+\sum_{p=0}^{n-2} F_{p} F_{n-2-p}^{2}+F_{n-2}-1 \\
& +\left\{\begin{aligned}
1 & \text { if } n \text { is odd } \\
-1 & \text { if } n \text { is even }
\end{aligned}\right. \\
& =\sum_{p=0}^{n} F_{p} F_{n-p}^{2}+F_{n-1}+F_{n-2}+\left\{\begin{aligned}
1 & \text { if } n \text { is odd } \\
-1 & \text { if } n \text { is even }
\end{aligned}\right. \\
& =\sum_{p=0}^{n} F_{p} F_{n-p}^{2}+F_{n}+\left\{\begin{aligned}
1 & \text { if } n \text { is odd } \\
-1 & \text { if } n \text { is even. }
\end{aligned}\right.
\end{aligned}
$$

Regarding repetitions of one letter, $R_{p} G(\varphi)=\left[\left(\begin{array}{l}1 \\ p\end{array}\right)+\left(\begin{array}{l}1 \\ p\end{array}\right)\left(\begin{array}{l}1 \\ p\end{array}\right)\right]$ and, for $n \geq 0$, the vector $R_{p} G\left(\varphi^{n+2}\right)$ is obtained from equation (5).

Corollary 3 For any integer $n \geq 0$,

$$
R_{p} G\left(\varphi^{n+2}\right)=\left[\begin{array}{cc}
\left(\begin{array}{c}
F_{n+2} \\
p
\end{array}\right)+\left(\begin{array}{c}
F_{n+1} \\
p
\end{array}\right) & \left(\begin{array}{c}
F_{n+1} \\
p
\end{array}\right)+\left(\begin{array}{c}
F_{n} \\
p
\end{array}\right)
\end{array}\right] .
$$

\section{A Particular Family of Morphisms}

Let $k$ be an integer $(k \geq 2)$ and $A$ the $k$-letter ordered alphabet $A=\left\{a_{1}<a_{2}<\cdots<a_{k}\right\}$. In this section we are interested in morphisms $f$ having the following particularities:

a) there exists a positive integer $m$ such that $\left|f\left(a_{1}\right)\right|_{a_{i}}=m, 1 \leq i \leq k$,

b) there exists a positive integer $d$ such that $\left|f\left(a_{2} \ldots a_{k}\right)\right|_{a_{i}}=d, 1 \leq i \leq k$,

c) for every $i, j, 1 \leq i, j \leq k,\left|f\left(a_{i} a_{j}\right)\right|_{1 \# 2}^{e x t}=\left|f\left(a_{j} a_{i}\right)\right|_{1 \# 2}^{e x t}$.

(Conditions (a) and (b) are particular cases of the more general situation, considered in Theorem 1 below, in which the alphabet $A$ is partitioned into sets $A_{1}, A_{2}, \ldots, A_{n}$ such that, for each $A_{i}$, the sum of the number of occurrences of each letter in the images of letters of $A_{i}$ is the same.)

In this case we are able to give direct formulas to compute $\left|f^{n+1}\left(a_{1}\right)\right|_{1 \# 2}$ and others from $m$, $d$, and $n$. 
Proposition 3 For every positive integer $n$,

$$
\begin{aligned}
& \left|f^{n+1}\left(a_{1}\right)\right|_{1 \# 2}=m(d+m)^{n-1} \sum_{i=1}^{k}\left|f\left(a_{i}\right)\right|_{1 \# 2}+\frac{\left[m(d+m)^{n-1}-1\right] m(d+m)^{n-1}}{2} \sum_{j=1}^{k}\left|f\left(a_{j} a_{j}\right)\right|_{1 \# 2}^{e x t} \\
& +m^{2}(d+m)^{2 n-2} \sum_{1 \leq i<j \leq k}\left|f\left(a_{i} a_{j}\right)\right|_{1 \# 2}^{e x t}, \\
& \left|f^{n+1}\left(a_{2} \ldots a_{k}\right)\right|_{1 \# 2}=d(d+m)^{n-1} \sum_{i=1}^{k}\left|f\left(a_{i}\right)\right|_{1 \# 2}+\frac{\left[d(d+m)^{n-1}-1\right] d(d+m)^{n-1}}{2} \sum_{j=1}^{k}\left|f\left(a_{j} a_{j}\right)\right|_{1 \# 2}^{e x t} \\
& +d^{2}(d+m)^{2 n-2} \sum_{1 \leq i<j \leq k}\left|f\left(a_{i} a_{j}\right)\right|_{1 \# 2}^{e x t} .
\end{aligned}
$$

Proof. Let $n \geq 1$. As in Proposition 1 , let $q_{1}=\left|f^{n}\left(a_{1}\right)\right|$ so that $f^{n}\left(a_{1}\right)=a_{1_{1}^{\prime}} \ldots a_{1_{q_{1}}^{\prime}}$. Equation (2) gives

$$
\begin{aligned}
\left|f^{n+1}\left(a_{1}\right)\right|_{1 \# 2} & =\sum_{1 \leq i<j \leq q_{1}}\left(\sum_{r=1}^{k-1}\left(m_{1, r, 1_{i}^{\prime}} \cdot \sum_{s=r+1}^{k} m_{1, s, 1_{j}^{\prime}}\right)\right)+\sum_{t=1}^{k}\left|f\left(a_{t}\right)\right|_{1 \# 2} \cdot m_{n, t, 1} \\
& =\sum_{1 \leq i<j \leq q_{1}}\left|f\left(a_{1_{i}^{\prime}} a_{1_{j}^{\prime}}\right)\right|_{1 \# 2}^{e x t}+\sum_{t=1}^{k}\left|f\left(a_{t}\right)\right|_{1 \# 2} \cdot\left|f^{n}\left(a_{1}\right)\right|_{a_{t}} .
\end{aligned}
$$

Now, conditions (a) to (c) above imply that the incidence matrix of $f^{n}$ is rather special. From (a) and (b), $\left|f^{n}\left(a_{1}\right)\right|_{a_{t}}=m(d+m)^{n-1}$ for each $t, 1 \leq t \leq k$. This implies that $\sum_{t=1}^{k}\left|f\left(a_{t}\right)\right|_{1 \# 2} \cdot\left|f^{n}\left(a_{1}\right)\right|_{a_{t}}=m(d+m)^{n-1} \sum_{i=1}^{k}\left|f\left(a_{i}\right)\right|_{1 \# 2}$. This also implies that $q_{1}=$ $k m(d+m)^{n-1}$.

But, from (c), the computation of $\sum_{1 \leq i<j \leq k m(d+m)^{n-1}}\left|f\left(a_{1_{i}^{\prime}} a_{1_{j}^{\prime}}\right)\right|_{1 \# 2}^{e x t}$, realized from the word $f^{n}\left(a_{1}\right)$ which contains $m(d+m)^{n-1}$ occurrences of each letter, can be equivalently realized from the word $a_{1}^{m(d+m)^{n-1}} a_{2}^{m(d+m)^{n-1}} \cdots a_{k}^{m(d+m)^{n-1}}$.

Then the first letter $a_{1}(i=1)$ gives $\left[m(d+m)^{n-1}-1\right] \cdot\left|f\left(a_{1} a_{1}\right)\right|_{1 \# 2}^{\text {ext }}+\sum_{j=2}^{k} m(d+$ $m)^{n-1}\left|f\left(a_{1} a_{j}\right)\right|_{1 \# 2}^{e x t}$.

The second letter $a_{1}(i=2)$ gives $\left[m(d+m)^{n-1}-2\right] \cdot\left|f\left(a_{1} a_{1}\right)\right|_{1 \# 2}^{\text {ext }}+\sum_{j=2}^{k} m(d+$ $m)^{n-1}\left|f\left(a_{1} a_{j}\right)\right|_{1 \# 2}^{e x t}$.

The next to last letter $a_{1}\left(i=m(d+m)^{n-1}-1\right)$ gives $1 \cdot\left|f\left(a_{1} a_{1}\right)\right|_{1 \# 2}^{\text {ext }}+\sum_{j=2}^{k} m(d+$ $m)^{n-1}\left|f\left(a_{1} a_{j}\right)\right|_{1 \# 2}^{e x t}$.

The last letter $a_{1}\left(i=m(d+m)^{n-1}\right)$ gives $0 \cdot\left|f\left(a_{1} a_{1}\right)\right|_{1 \# 2}^{e x t}+\sum_{j=2}^{k} m(d+m)^{n-1}\left|f\left(a_{1} a_{j}\right)\right|_{1 \# 2}^{e x t}$.

The first letter $a_{2}\left(i=m(d+m)^{n-1}+1\right)$ gives $\left[m(d+m)^{n-1}-1\right] \cdot\left|f\left(a_{2} a_{2}\right)\right|_{1 \# 2}^{\text {ext }}+$ $\sum_{j=3}^{k} m(d+m)^{n-1}\left|f\left(a_{2} a_{j}\right)\right|_{1 \# 2}^{e x t}$.

And so on. 
Consequently $\sum_{1 \leq i<j \leq k m(d+m)^{n-1}}\left|f\left(a_{1_{i}^{\prime}} a_{1_{j}^{\prime}}\right)\right|_{1 \# 2}^{e x t}$

$$
\begin{aligned}
& =\sum_{i=0}^{m(d+m)^{n-1}-1} i \cdot \sum_{j=1}^{k}\left|f\left(a_{j} a_{j}\right)\right|_{1 \# 2}^{e x t}+m(d+m)^{n-1} \sum_{i=1}^{k-1} \sum_{j=i+1}^{k} m(d+m)^{n-1}\left|f\left(a_{i} a_{j}\right)\right|_{1 \# 2}^{e x t} \\
& =\frac{\left[m(d+m)^{n-1}-1\right] m(d+m)^{n-1}}{2} \sum_{j=1}^{k}\left|f\left(a_{j} a_{j}\right)\right|_{1 \# 2}^{e x t}+m^{2}(d+m)^{2 n-2} \sum_{1 \leq i<j \leq k}\left|f\left(a_{i} a_{j}\right)\right|_{1 \# 2}^{e x t} .
\end{aligned}
$$

Hence, we have

$$
\begin{aligned}
\left|f^{n+1}\left(a_{1}\right)\right|_{1 \# 2} & =m(d+m)^{n-1} \sum_{i=1}^{k}\left|f\left(a_{i}\right)\right|_{1 \# 2} \\
& +\frac{\left[m(d+m)^{n-1}-1\right] m(d+m)^{n-1}}{2} \sum_{j=1}^{k}\left|f\left(a_{j} a_{j}\right)\right|_{1 \# 2}^{e x t} \\
& +m^{2}(d+m)^{2 n-2} \sum_{1 \leq i<j \leq k}\left|f\left(a_{i} a_{j}\right)\right|_{1 \# 2}^{e x t} .
\end{aligned}
$$

Now, for $\left|f^{n+1}\left(a_{2} \ldots a_{k}\right)\right|_{1 \# 2}$, equation (2) gives

$$
\begin{aligned}
\left|f^{n+1}\left(a_{2} \ldots a_{k}\right)\right|_{1 \# 2} & =\sum_{\ell=2}^{k}\left(\sum_{1 \leq i<j \leq q_{\ell}}\left|f\left(a_{\ell_{i}^{\prime}} a_{\ell_{j}^{\prime}}\right)\right|_{1 \# 2}^{e x t}+\sum_{t=1}^{k}\left|f\left(a_{t}\right)\right|_{1 \# 2} \cdot\left|f^{n}\left(a_{\ell}\right)\right|_{a_{t}}\right) \\
& =\sum_{\ell=2}^{k} \sum_{1 \leq i<j \leq q_{\ell}}\left|f\left(a_{\ell_{i}^{\prime}} a_{\ell_{j}^{\prime}}\right)\right|_{1 \# 2}^{e x t}+\sum_{t=1}^{k} \sum_{\ell=2}^{k}\left|f\left(a_{t}\right)\right|_{1 \# 2} \cdot\left|f^{n}\left(a_{\ell}\right)\right|_{a_{t}} .
\end{aligned}
$$

Again from (a) and (b), $\sum_{\ell=2}^{k}\left|f^{n}\left(a_{\ell}\right)\right|_{a_{t}}=d(d+m)^{n-1}$ for each $t, 1 \leq t \leq k$. In particular, $\sum_{\ell=2}^{k} \sum_{1 \leq i<j \leq q \ell}\left|f\left(a_{\ell_{i}^{\prime}} a_{\ell_{j}^{\prime}}\right)\right|_{1 \# 2}^{e x t}=\sum_{1 \leq i<j \leq k d(d+m)^{n-1}}\left|f\left(a_{\ell_{i}^{\prime}} a_{\ell_{j}^{\prime}}\right)\right|_{1 \# 2}^{e x t}$. As above, the computation can be realized from the word $a_{1}^{d(d+m)^{n-1}} a_{2}^{d(d+m)^{n-1}} \cdots a_{k}^{d(d+m)^{n-1}}$. This gives us $\sum_{1 \leq i<j \leq k d(d+m)^{n-1}}\left|f\left(a_{\ell_{i}^{\prime}} a_{\ell_{j}^{\prime}}\right)\right|_{1 \# 2}^{e x t}$

$$
\begin{aligned}
& =\sum_{i=0}^{d(d+m)^{n-1}-1} i \cdot \sum_{j=1}^{k}\left|f\left(a_{j} a_{j}\right)\right|_{1 \# 2}^{e x t}+d(d+m)^{n-1} \sum_{i=1}^{k-1} \sum_{j=i+1}^{k} d(d+m)^{n-1}\left|f\left(a_{i} a_{j}\right)\right|_{1 \# 2}^{e x t} \\
& =\frac{\left[d(d+m)^{n-1}-1\right] d(d+m)^{n-1}}{2} \sum_{j=1}^{k}\left|f\left(a_{j} a_{j}\right)\right|_{1 \# 2}^{e x t}+d^{2}(d+m)^{2 n-2} \sum_{1 \leq i<j \leq k}\left|f\left(a_{i} a_{j}\right)\right|_{1 \# 2}^{e x t} .
\end{aligned}
$$

Now the same reasoning can be applied for $\left|f^{n+1}\left(a_{1}\right)\right|_{2 \# 1}$ and $\left|f^{n+1}\left(a_{2} \ldots a_{k}\right)\right|_{2 \# 1}$, because of the following obvious property.

Property 3 Let $f$ be a morphism on A. For every non-negative integer $n$, and for every integers $i, j, 1 \leq i, j \leq k,\left|f^{n}\left(a_{i} a_{j}\right)\right|_{1 \# 2}^{e x t}=\left|f^{n}\left(a_{j} a_{i}\right)\right|_{2 \# 1}^{e x t}$.

Thus, using equation (4), we have the following.

Proposition 4 For every positive integer n,

$$
\begin{gathered}
\left|f^{n+1}\left(a_{1}\right)\right|_{2 \# 1}=m(d+m)^{n-1} \sum_{i=1}^{k}\left|f\left(a_{i}\right)\right|_{2 \# 1}+\frac{\left[m(d+m)^{n-1}-1\right] m(d+m)^{n-1}}{2} \sum_{j=1}^{k}\left|f\left(a_{j} a_{j}\right)\right|_{2 \# 1}^{\text {ext }} \\
+m^{2}(d+m)^{2 n-2} \sum_{1 \leq i<j \leq k}\left|f\left(a_{i} a_{j}\right)\right|_{2 \# 1}^{e x t}, \\
\left|f^{n+1}\left(a_{2} \ldots a_{k}\right)\right|_{2 \# 1}=d(d+m)^{n-1} \sum_{i=1}^{k}\left|f\left(a_{i}\right)\right|_{2 \# 1}+\frac{\left[d(d+m)^{n-1}-1\right] d(d+m)^{n-1}}{2} \sum_{j=1}^{k}\left|f\left(a_{j} a_{j}\right)\right|_{2 \# 1}^{\text {ext }} \\
+d^{2}(d+m)^{2 n-2} \sum_{1 \leq i<j \leq k}\left|f\left(a_{i} a_{j}\right)\right|_{2 \# 1}^{e x t} .
\end{gathered}
$$


The previous reasoning can of course be applied if conditions (a) and (b) are verified for any partition of the alphabet (in Propositions 3 and 4 the partition is in two sets $A=$ $\left.\left\{a_{1}\right\} \cup\left\{a_{2} \ldots a_{k}\right\}\right)$. Then we obtain the following general result.

Theorem 1 Let $k$ be an integer $(k \geq 2)$, and $A$ the $k$-letter ordered alphabet $A=\left\{a_{1}<\right.$ $\left.a_{2}<\ldots<a_{k}\right\}$. Let $f$ be a morphism on A fulfilling the following conditions:

- there exist a positive integer $p$ and a set of p positive integers $\left\{m_{1}, \ldots, m_{p}\right\}$ such that $A$ can be partitioned into $p$ subsets $A_{1}, \ldots, A_{p}$ with $\sum_{a \in A_{\ell}}|f(a)|_{a_{i}}=m_{\ell}, 1 \leq i \leq k$,

- for every $i, j, 1 \leq i, j \leq k,\left|f\left(a_{i} a_{j}\right)\right|_{1 \# 2}^{e x t}=\left|f\left(a_{j} a_{i}\right)\right|_{1 \# 2}^{e x t}$.

Let $M=m_{1}+\ldots+m_{p}$ and let $u=1 \# 2$ or $u=2 \# 1$. Then, for every positive integer $n$ and for each $A_{\ell}, 1 \leq \ell \leq p$,

$$
\begin{aligned}
\sum_{a \in A_{\ell}}\left|f^{n+1}(a)\right|_{u}= & m_{\ell} M^{n-1} \sum_{i=1}^{k}\left|f\left(a_{i}\right)\right|_{u}+\frac{\left(m_{\ell} M^{n-1}-1\right) m_{\ell} M^{n-1}}{2} \sum_{j=1}^{k}\left|f\left(a_{j} a_{j}\right)\right|_{u}^{e x t} \\
& +m_{\ell}^{2} M^{2 n-2} \sum_{1 \leq i<j \leq k}\left|f\left(a_{i} a_{j}\right)\right|_{u}^{e x t} .
\end{aligned}
$$

\section{Examples}

In this section we give a series of examples of application of Theorem 1 . The first ones (5.1 to 5.4) are related to the well known Thue-Morse morphism and they give results that of course can be found with some other techniques, but they are presented in order to make the results more comprehensible. The last ones are original; they illustrate some general representative situations.

\subsection{The Thue-Morse Morphism}

The Thue-Morse morphism (see Section 3.2) is the simplest example of a morphism fulfilling conditions (a) to (c) above. Indeed $m=d=1$, and $\left|\mu\left(a_{1} a_{2}\right)\right|_{1 \# 2}^{\text {ext }}=\left|a_{1} a_{2} a_{2} a_{1}\right|_{1 \# 2}^{\text {ext }}=$ $1=\left|a_{2} a_{1} a_{1} a_{2}\right|_{1 \# 2}^{e x t}=\left|\mu\left(a_{2} a_{1}\right)\right|_{1 \# 2}^{e x t},\left|\mu\left(a_{1} a_{1}\right)\right|_{1 \# 2}^{e x t}=\left|\mu\left(a_{2} a_{2}\right)\right|_{1 \# 2}^{e x t}=1$. Since $\left|\mu\left(a_{1}\right)\right|_{1 \# 2}=$ $\left|\mu\left(a_{2}\right)\right|_{2 \# 1}=1$, and $\left|\mu\left(a_{1}\right)\right|_{2 \# 1}=\left|\mu\left(a_{2}\right)\right|_{1 \# 2}=0$, we obtain from Propositions 3 and 4 that $\left|\mu^{n+1}\left(a_{1}\right)\right|_{1 \# 2}=\left|\mu^{n+1}\left(a_{1}\right)\right|_{2 \# 1}=\left|\mu^{n+1}\left(a_{2}\right)\right|_{1 \# 2}=\left|\mu^{n+1}\left(a_{2}\right)\right|_{2 \# 1}=2^{2 n-1}$ (see Corollary 1 above).

\subsection{The Istrail Morphism}

In [15] we have the following well known example of a weakly square-free morphism. The morphism $h$ is defined on the three-letter ordered alphabet $A=\left\{a_{1}<a_{2}<a_{3}\right\}$ by

$$
h\left(a_{1}\right)=a_{1} a_{2} a_{3}, \quad h\left(a_{2}\right)=a_{1} a_{3}, \quad h\left(a_{3}\right)=a_{2}
$$


(we remark that $h$ generates a square-free infinite word, $h^{\omega}\left(a_{1}\right)$, but is not a square-free morphism: $h\left(a_{1} a_{2} a_{1}\right)=a_{1} a_{2} a_{3} a_{1} a_{3} a_{1} a_{2} a_{3}$ contains the square $\left.a_{3} a_{1} a_{3} a_{1}\right)$.

The word $h^{\omega}\left(a_{1}\right)$ is closely related to the Thue-Morse word $\mathbf{t}$. Indeed, let $B$ be the two-letter alphabet $B=\left\{a_{1}, a_{2}\right\}$, and consider the morphism

$$
\begin{aligned}
\delta: A^{*} & \rightarrow B^{*} \\
a_{1} & \mapsto a_{1} \\
a_{2} & \mapsto a_{1} a_{2} \\
a_{3} & \mapsto a_{1} a_{2} a_{2}
\end{aligned}
$$

Then $\mathbf{t}=\delta\left(h^{\omega}\left(a_{1}\right)\right)$ (see Proposition 2.3.2 in [21]).

Here again the morphism $h$ fulfills conditions (a) to (c) with $m=d=1$. Moreover $\left|h\left(a_{1}\right)\right|_{1 \# 2}=\left|h\left(a_{1} a_{1}\right)\right|_{1 \# 2}^{e x t}=3,\left|h\left(a_{2}\right)\right|_{1 \# 2}=\left|h\left(a_{2} a_{2}\right)\right|_{1 \# 2}^{e x t}=1,\left|h\left(a_{3}\right)\right|_{1 \# 2}=\left|h\left(a_{3} a_{3}\right)\right|_{1 \# 2}^{e x t}=0$, and $\left|h\left(a_{1} a_{2}\right)\right|_{1 \# 2}^{e x t}=2,\left|h\left(a_{1} a_{3}\right)\right|_{1 \# 2}^{e x t}=\left|h\left(a_{2} a_{3}\right)\right|_{1 \# 2}^{\text {ext }}=1$. Then, from Proposition 3 , for every integer $n \geq 1,\left|h^{n+1}\left(a_{1}\right)\right|_{1 \# 2}=\left|h^{n+1}\left(a_{2} a_{3}\right)\right|_{1 \# 2}=3 \cdot 2^{2 n-1}+2^{n}$.

From Property 3, the values for $2 \# 1$ are the same as for $1 \# 2$, except for $\left|h\left(a_{i}\right)\right|_{2 \# 1}$, $1 \leq i \leq 3$. Here $\left|h\left(a_{1}\right)\right|_{2 \# 1}=\left|h\left(a_{2}\right)\right|_{2 \# 1}=\left|h\left(a_{3}\right)\right|_{2 \# 1}=0$. Thus, from Proposition 4 , for every integer $n \geq 1,\left|h^{n+1}\left(a_{1}\right)\right|_{2 \# 1}=\left|h^{n+1}\left(a_{2} a_{3}\right)\right|_{2 \# 1}=3 \cdot 2^{2 n-1}-2^{n}$.

\subsection{The Prouhet morphisms}

In 1851, Prouhet [25] gave an algorithm to realize an arithmetic construction. This algorithm produces intermediate infinite words that are a generalization of the Thue-Morse word (see above). It was proved in [28] that these words can be generated by morphisms (see also [1]).

Let $k \geq 2$, and let $A$ be the $k$-letter ordered alphabet $A=\left\{a_{1}<\cdots<a_{k}\right\}$. The Prouhet morphism $\pi_{k}$ is defined on $A$ by $\pi_{k}\left(a_{i}\right)=a_{i} a_{i+1} \ldots a_{k} a_{1} \ldots a_{i-1}, 1 \leq i \leq k$.

Example. Let $k=6$. The morphism $\pi_{6}$ is given by

$$
\begin{aligned}
a_{1} & \mapsto a_{1} a_{2} a_{3} a_{4} a_{5} a_{6} \\
a_{2} & \mapsto a_{2} a_{3} a_{4} a_{5} a_{6} a_{1} \\
a_{3} & \mapsto a_{3} a_{4} a_{5} a_{6} a_{1} a_{2} \\
a_{4} & \mapsto a_{4} a_{5} a_{6} a_{1} a_{2} a_{3} \\
a_{5} & \mapsto a_{5} a_{6} a_{1} a_{2} a_{3} a_{4} \\
a_{6} & \mapsto a_{6} a_{1} a_{2} a_{3} a_{4} a_{5} .
\end{aligned}
$$

For every $k$ the morphism $\pi_{k}$ fulfills the conditions of Theorem 1. Since, for every $i$, $1 \leq i \leq k$, the word $\pi_{k}\left(a_{i}\right)$ contains exactly one occurrence of each letter of $A$, there are a lot of possibilities to choose the partition of $A$. Here we choose $p=k$ and $A=A_{1} \cup \ldots \cup A_{k}$, $A_{i}=\left\{a_{i}\right\}, 1 \leq i \leq k$. This implies that $m_{i}=1,1 \leq i \leq k$ and, of course, $M=k$.

Also, for every $i, j, 1 \leq i, j \leq k,\left|\pi_{k}\left(a_{i} a_{j}\right)\right|_{1 \# 2}^{e x t}=\left|\pi_{k}\left(a_{i} a_{j}\right)\right|_{2 \# 1}^{e x t}=\frac{k(k-1)}{2}$. 
Now it is easy to verify that, due to the particular form of the images of the letters by $\pi_{k}$, one has for every $\ell, 1 \leq \ell \leq k,\left|\pi_{k}\left(a_{\ell}\right)\right|_{1 \# 2}=\frac{[k-(\ell-1)](k-\ell)}{2}+\frac{(\ell-1)(\ell-2)}{2}$ and $\left|\pi_{k}\left(a_{\ell}\right)\right|_{2 \# 1}=$ $(\ell-1)[k-(\ell-1)]$.

Thus we obtain the following corollary of Theorem 1.

Corollary 4 For every $i, 1 \leq i \leq k$, and for every positive integer $n$,

$$
\begin{aligned}
\left|\pi_{k}^{n+1}\left(a_{i}\right)\right|_{1 \# 2} & =\frac{(k-1) k^{n}}{12}\left(3 k^{n+1}+k-2\right), \\
\left|\pi_{k}^{n+1}\left(a_{i}\right)\right|_{2 \# 1} & =\frac{(k-1) k^{n}}{12}\left(3 k^{n+1}-k+2\right) .
\end{aligned}
$$

Proof. From Theorem 1 and from what precedes,

$$
\begin{aligned}
\left|\pi_{k}^{n+1}\left(a_{i}\right)\right|_{1 \# 2}= & k^{n-1} \cdot \sum_{\ell=1}^{k}\left(\frac{[k-(\ell-1)](k-\ell)}{2}+\frac{(\ell-1)(\ell-2)}{2}\right)+\frac{\left(k^{n-1}-1\right) k^{n-1}}{2} \sum_{j=1}^{k} \frac{k(k-1)}{2} \\
& +k^{2 n-2} \cdot \sum_{1 \leq j<\ell \leq k} \frac{k(k-1)}{2} \\
= & k^{n-1} \cdot\left[\sum_{\ell=1}^{k}\left(\frac{[k-(\ell-1)](k-\ell)}{2}+\frac{(\ell-1)(\ell-2)}{2}\right)+\frac{\left(k^{n-1}-1\right)}{2} \cdot \frac{k^{2}(k-1)}{2}\right. \\
& \left.\quad k^{n-1} \cdot\left[\frac{k(k-1)}{2}\right]^{2}\right] .
\end{aligned}
$$

Since $\sum_{\ell=1}^{k}[k-(\ell-1)](k-\ell)=\frac{k^{3}-k}{3}$ and $\sum_{\ell=1}^{k}(\ell-1)(\ell-2)=\frac{k}{3}(k-2)(k-1)$, we obtain

$$
\begin{aligned}
\left|\pi_{k}^{n+1}\left(a_{i}\right)\right|_{1 \# 2}= & k^{n-1} \cdot\left[\frac{(k+1)}{6} k(k-1)+\frac{(k-2)}{6} k(k-1)+\frac{k\left(k^{n-1}-1\right)}{4} k(k-1)\right. \\
& \left.\quad+\frac{k^{n-1} k(k-1)}{4} k(k-1)\right] \\
= & \frac{(k-1) k^{n}}{12}\left(3 k^{n+1}+k-2\right)
\end{aligned}
$$

The proof is the same for $\left|\pi_{k}^{n+1}\left(a_{i}\right)\right|_{2 \# 1}$, using $\sum_{\ell=1}^{k}(\ell-1)[k-(\ell-1)]=\frac{k}{6}\left(k^{2}-1\right)$.

Example (continued).

$$
\begin{aligned}
\left|\pi_{6}\left(a_{1}\right)\right|_{1 \# 2}=15, & \left|\pi_{6}\left(a_{1}\right)\right|_{2 \# 1}=0 \\
\left|\pi_{6}\left(a_{2}\right)\right|_{1 \# 2}=10, & \left|\pi_{6}\left(a_{2}\right)\right|_{2 \# 1}=5, \\
\left|\pi_{6}\left(a_{3}\right)\right|_{1 \# 2}=7, & \left|\pi_{6}\left(a_{3}\right)\right|_{2 \# 1}=8 \\
\left|\pi_{6}\left(a_{4}\right)\right|_{1 \# 2}=6, & \left|\pi_{6}\left(a_{4}\right)\right|_{2 \# 1}=9 \\
\left|\pi_{6}\left(a_{5}\right)\right|_{1 \# 2}=7, & \left|\pi_{6}\left(a_{5}\right)\right|_{2 \# 1}=8 \\
\left|\pi_{6}\left(a_{6}\right)\right|_{1 \# 2}=10, & \left|\pi_{6}\left(a_{6}\right)\right|_{2 \# 1}=5 .
\end{aligned}
$$


Here, $k=6$, so for every $i, 1 \leq i \leq k$, and for every $n \geq 1$,

$$
\begin{aligned}
\left|\pi_{6}^{n+1}\left(a_{i}\right)\right|_{1 \# 2} & =\frac{5 \cdot 6^{n}}{12}\left(3 \cdot 6^{n+1}+6-2\right) \\
& =6^{n-1} \cdot\left(45 \cdot 6^{n}+10\right), \\
\left|\pi_{6}^{n+1}\left(a_{i}\right)\right|_{2 \# 1} & =6^{n-1} \cdot\left(45 \cdot 6^{n}-10\right) .
\end{aligned}
$$

\subsection{The Arshon Morphisms}

In a paper written in 1937, Arshon [3] gives an algorithm to construct for each integer $n$, $n \geq 3$, an infinite square-free word over an $n$-letter alphabet, and in the case of two letters a cube-free word. It appears now that this construction is closely connected to the use of Prouhet morphisms. In the case of two letters the Arshon word is the Thue-Morse word and Arshon's algorithm gives exactly the Thue-Morse morphism which is a particular case of Prouhet morphism.

The Arshon words were proved to be, in the odd case, an example of infinite words that can be generated by a tag-system but not by a morphism (see [5], [16], and [13]). In [29] a family of morphisms is given which generates the even case Arshon words (see also [13] and $[16])$. These morphisms are the following.

Let $k$ be any even positive integer. The morphism $\beta_{k}$ is defined, for every $r, 1 \leq r \leq k / 2$, by

$$
\begin{array}{ll}
a_{2 r-1} & \mapsto a_{2 r-1} a_{2 r} \ldots a_{k-1} a_{k} a_{1} a_{2} \ldots a_{2 r-3} a_{2 r-2} \\
a_{2 r} & \mapsto a_{2 r-1} a_{2 r-2} \ldots a_{2} a_{1} a_{k} a_{k-1} \ldots a_{2 r+1} a_{2 r}
\end{array}
$$

(We remark that, again, though they generate square-free infinite words, the morphisms $\beta_{k}$ are not square-free morphisms.)

Example. Let $k=6$. The morphism $\beta_{6}$ is given by

$$
\begin{aligned}
& a_{1} \mapsto a_{1} a_{2} a_{3} a_{4} a_{5} a_{6} \\
& a_{2} \mapsto a_{1} a_{6} a_{5} a_{4} a_{3} a_{2} \\
& a_{3} \mapsto a_{3} a_{4} a_{5} a_{6} a_{1} a_{2} \\
& a_{4} \mapsto a_{3} a_{2} a_{1} a_{6} a_{5} a_{4} \\
& a_{5} \mapsto a_{5} a_{6} a_{1} a_{2} a_{3} a_{4} \\
& a_{6} \mapsto a_{5} a_{4} a_{3} a_{2} a_{1} a_{6}
\end{aligned}
$$

Of course, since it is obtained from $\pi_{k}$ in an obvious manner, the morphism $\beta_{k}$ fulfills the conditions of Theorem 1 for every even $k$. Since, for every $i, 1 \leq i \leq k$, the word $\beta_{k}\left(a_{i}\right)$ contains exactly one occurrence of each letter of $A$, there are again a lot of possibilities to choose the partition of $A$. Here we again choose $p=k$ and $A=A_{1} \cup \ldots \cup A_{k}, A_{i}=\left\{a_{i}\right\}$, $1 \leq i \leq k$. This implies that $m_{i}=1,1 \leq i \leq k$ and, of course, $M=k$.

Also, for every $i, j, 1 \leq i, j \leq k,\left|\beta_{k}\left(a_{i} a_{j}\right)\right|_{1 \# 2}^{e x t}=\left|\beta_{k}\left(a_{i} a_{j}\right)\right|_{2 \# 1}^{e x t}=\frac{k(k-1)}{2}$. 
Now, again because $\beta_{k}$ is directly obtained from $\pi_{k}$, one has for every $r, 1 \leq r \leq \frac{k}{2}$,

$$
\begin{aligned}
\left|\beta_{k}\left(a_{2 r-1}\right)\right|_{1 \# 2} & =\frac{[k-(2 r-2)][k-(2 r-1)]}{2}+\frac{(2 r-2)(2 r-3)}{2}, \\
\left|\beta_{k}\left(a_{2 r}\right)\right|_{1 \# 2} & =(2 r-1)[k-(2 r-1)] \\
\left|\beta_{k}\left(a_{2 r-1}\right)\right|_{2 \# 1} & =(2 r-2)[k-(2 r-2)] \\
\left|\beta_{k}\left(a_{2 r}\right)\right|_{2 \# 1} & =\frac{[k-(2 r-1)](k-2 r)}{2}+\frac{(2 r-1)(2 r-2)}{2} .
\end{aligned}
$$

Thus, we obtain another corollary of Theorem 1.

Corollary 5 Let $k$ be any even positive integer. For every $i, 1 \leq i \leq k$, and for every positive integer $n$,

$$
\begin{aligned}
& \left|\beta_{k}^{n+1}\left(a_{i}\right)\right|_{1 \# 2}=\frac{k^{n-1}}{4}\left[k^{n+2} \cdot(k-1)+2 k\right], \\
& \left|\beta_{k}^{n+1}\left(a_{i}\right)\right|_{2 \# 1}=\frac{k^{n-1}}{4}\left[k^{n+2} \cdot(k-1)-2 k\right] .
\end{aligned}
$$

Proof. As for the proof of Corollary 4, we obtain from Theorem 1 and from what precedes,

$$
\left|\beta_{k}^{n+1}\left(a_{i}\right)\right|_{1 \# 2}=k^{n-1} \cdot\left[\sum_{\ell=1}^{k}\left|\beta_{k}\left(a_{\ell}\right)\right|_{1 \# 2}-\frac{k^{2}(k-1)}{4}\right]+k^{n-1} \cdot\left[\frac{k^{n+2} \cdot(k-1)}{4}\right] .
$$

But $\sum_{\ell=1}^{k}\left|\beta_{k}\left(a_{\ell}\right)\right|_{1 \# 2}=\sum_{r=1}^{k / 2}\left[\left|\beta_{k}\left(a_{2 r-1}\right)\right|_{1 \# 2}+\left|\beta_{k}\left(a_{2 r}\right)\right|_{1 \# 2}\right]=\frac{k^{2}(k-1)}{4}+\frac{k}{2}$, and the result follows. The proof is the same for $\left|\beta_{k}^{n+1}\left(a_{i}\right)\right|_{2 \# 1}$, using $\sum_{\ell=1}^{k}\left|\beta_{k}\left(a_{\ell}\right)\right|_{2 \# 1}=\frac{k^{2}(k-1)}{4}-\frac{k}{2}$.

Example (continued).

$$
\begin{array}{lrlrl}
\left|\beta_{6}\left(a_{1}\right)\right|_{1 \# 2}=15, & \left|\beta_{6}\left(a_{1}\right)\right|_{2 \# 1}=0, \\
\left|\beta_{6}\left(a_{2}\right)\right|_{1 \# 2}=5, & \left|\beta_{6}\left(a_{2}\right)\right|_{2 \# 1}=10, \\
\left|\beta_{6}\left(a_{3}\right)\right|_{1 \# 2}=7, & \left|\beta_{6}\left(a_{3}\right)\right|_{2 \# 1}=8, \\
\left|\beta_{6}\left(a_{4}\right)\right|_{1 \# 2}=9, & \left|\beta_{6}\left(a_{4}\right)\right|_{2 \# 1}=6, \\
\left|\beta_{6}\left(a_{5}\right)\right|_{1 \# 2}=7, & \left|\beta_{6}\left(a_{5}\right)\right|_{2 \# 1}=8, \\
\left|\beta_{6}\left(a_{6}\right)\right|_{1 \# 2}=5, & \left|\beta_{6}\left(a_{6}\right)\right|_{2 \# 1}=10 .
\end{array}
$$

For every $i, 1 \leq i \leq k$, and for every $n \geq 1$,

$$
\begin{aligned}
\left|\beta_{6}^{n+1}\left(a_{i}\right)\right|_{1 \# 2} & =\frac{6^{n-1}}{4} \cdot\left(6^{n+2} \cdot 5+2 \cdot 6\right) \\
& =6^{n-1} \cdot\left(45 \cdot 6^{n}+3\right), \\
\left|\beta_{6}^{n+1}\left(a_{i}\right)\right|_{2 \# 1} & =6^{n-1} \cdot\left(45 \cdot 6^{n}-3\right) .
\end{aligned}
$$




\subsection{Three Other Examples}

To end this list of examples, we give three morphisms that fulfill the conditions of Theorem 1, but are not linked with the Thue-Morse morphism. Moreover they are interesting because the first one is an erasing morphism, the second gives a non trivial partition of the alphabet when applying Theorem 1, and the third is an example of a ternary square-free morphism fulfilling the conditions.

1. Let $A$ be the four-letter ordered alphabet $A=\left\{a_{1}<a_{2}<a_{3}<a_{4}\right\}$. Define the morphism $f$ by

$$
\begin{aligned}
f: A^{*} & \rightarrow A^{*} \\
a_{1} & \mapsto a_{1} a_{3} a_{2} a_{4} \\
a_{2} & \mapsto \varepsilon \\
a_{3} & \mapsto a_{1} a_{4} \\
a_{4} & \mapsto a_{2} a_{3} .
\end{aligned}
$$

The morphism $f$ fulfills the conditions of Theorem 1 . Here we choose $p=3, A=$ $A_{1} \cup A_{2} \cup A_{3}$ with $A_{1}=\left\{a_{1}\right\}, A_{2}=\left\{a_{2}\right\}, A_{3}=\left\{a_{3}, a_{4}\right\}$, and $m_{1}=m_{3}=1, m_{2}=0$ (thus $M=2$ ). One has $\left|f\left(a_{1}\right)\right|_{1 \# 2}=5,\left|f\left(a_{3}\right)\right|_{1 \# 2}=\left|f\left(a_{4}\right)\right|_{1 \# 2}=1,\left|f\left(a_{1}\right)\right|_{2 \# 1}=1$, $\left|f\left(a_{3}\right)\right|_{2 \# 1}=\left|f\left(a_{4}\right)\right|_{2 \# 1}=0,\left|f\left(a_{1} a_{1}\right)\right|_{1 \# 2}^{e x t}=\left|f\left(a_{1} a_{1}\right)\right|_{2 \# 1}^{e x t}=6,\left|f\left(a_{3} a_{3}\right)\right|_{1 \# 2}^{e x t}=\left|f\left(a_{3} a_{3}\right)\right|_{2 \# 1}^{e x t}=$ $\left|f\left(a_{4} a_{4}\right)\right|_{1 \# 2}^{e x t}=\left|f\left(a_{4} a_{4}\right)\right|_{2 \# 1}^{e x t}=1,\left|f\left(a_{1} a_{3}\right)\right|_{1 \# 2}^{e x t}=\left|f\left(a_{1} a_{3}\right)\right|_{2 \# 1}^{e x t}=\left|f\left(a_{1} a_{4}\right)\right|_{1 \# 2}^{e x t}=\left|f\left(a_{1} a_{4}\right)\right|_{2 \# 1}^{e x t}=$ $3,\left|f\left(a_{3} a_{4}\right)\right|_{1 \# 2}^{e x t}=\left|f\left(a_{3} a_{4}\right)\right|_{2 \# 1}^{e x t}=2$. All the values with $a_{2}$ are of course 0 .

Then we have the following corollary of Theorem 1.

Corollary 6 For every positive integer $n$,

$$
\begin{aligned}
& \left|f^{n+1}\left(a_{1}\right)\right|_{1 \# 2}=\left|f^{n+1}\left(a_{3} a_{4}\right)\right|_{1 \# 2}=3 \cdot 2^{n-1} \cdot\left(2^{n+1}+1\right), \\
& \left|f^{n+1}\left(a_{1}\right)\right|_{2 \# 1}=\left|f^{n+1}\left(a_{3} a_{4}\right)\right|_{2 \# 1}=3 \cdot 2^{n-1} \cdot\left(2^{n+1}-1\right), \\
& \left|f^{n+1}\left(a_{2}\right)\right|_{1 \# 2}=\left|f^{n+1}\left(a_{2}\right)\right|_{2 \# 1}=0 .
\end{aligned}
$$

2. Let $A$ be the five-letter ordered alphabet $A=\left\{a_{1}<a_{2}<a_{3}<a_{4}<a_{5}\right\}$. Define the morphism $g$ by

$$
\begin{aligned}
g: A^{*} & \rightarrow A^{*} \\
a_{1} & \mapsto a_{1} a_{3} a_{5} a_{4} a_{2} \\
a_{2} & \mapsto a_{4} a_{2} a_{3} \\
a_{3} & \mapsto a_{5} a_{1} \\
a_{4} & \mapsto a_{1} a_{5} \\
a_{5} & \mapsto a_{2} a_{3} a_{4} .
\end{aligned}
$$

The morphism $g$ fulfills the conditions of Theorem 1 . Here we choose $p=3, A=$ $A_{1} \cup A_{2} \cup A_{3}$ with $A_{1}=\left\{a_{1}\right\}, A_{2}=\left\{a_{2}, a_{4}\right\}, A_{3}=\left\{a_{3}, a_{5}\right\}$, and $m_{1}=m_{2}=m_{3}=1$ (thus $M=3$ ). One has $\left|g\left(a_{1}\right)\right|_{1 \# 2}=6,\left|g\left(a_{2}\right)\right|_{1 \# 2}=\left|g\left(a_{4}\right)\right|_{1 \# 2}=1,\left|g\left(a_{3}\right)\right|_{1 \# 2}=0$, 
$\left|g\left(a_{5}\right)\right|_{1 \# 2}=3,\left|g\left(a_{1}\right)\right|_{2 \# 1}=4,\left|g\left(a_{2}\right)\right|_{2 \# 1}=2,\left|g\left(a_{3}\right)\right|_{2 \# 1}=1,\left|g\left(a_{4}\right)\right|_{2 \# 1}=\left|g\left(a_{5}\right)\right|_{2 \# 1}=$ $0,\left|g\left(a_{1} a_{1}\right)\right|_{1 \# 2}^{\text {ext }}=10,\left|g\left(a_{2} a_{2}\right)\right|_{1 \# 2}^{\text {ext }}=\left|g\left(a_{5} a_{5}\right)\right|_{1 \# 2}^{\text {ext }}=3,\left|g\left(a_{3} a_{3}\right)\right|_{1 \# 2}^{e x t}=\left|g\left(a_{4} a_{4}\right)\right|_{1 \# 2}^{e x t}=1$, $\left|g\left(a_{1} a_{2}\right)\right|_{1 \# 2}^{e x t}=\left|g\left(a_{1} a_{5}\right)\right|_{1 \# 2}^{e x t}=6,\left|g\left(a_{1} a_{3}\right)\right|_{1 \# 2}^{e x t}=\left|g\left(a_{1} a_{4}\right)\right|_{1 \# 2}^{e x t}=4,\left|g\left(a_{2} a_{3}\right)\right|_{1 \# 2}^{e x t}=\left|g\left(a_{2} a_{4}\right)\right|_{1 \# 2}^{e x t}=$ $\left|g\left(a_{2} a_{5}\right)\right|_{1 \# 2}^{e x t}=\left|g\left(a_{3} a_{5}\right)\right|_{1 \# 2}^{e x t}=\left|g\left(a_{4} a_{5}\right)\right|_{1 \# 2}^{e x t}=3,\left|g\left(a_{3} a_{4}\right)\right|_{1 \# 2}^{e x t}=1$.

To end we recall that, with the second condition of Theorem 1 and property 3 , one has for all integers $i, j, 1 \leq i, j \leq k,\left|g\left(a_{i} a_{j}\right)\right|_{1 \# 2}^{e x t}=\left|g\left(a_{i} a_{j}\right)\right|_{2 \# 1}^{e x t}$.

Then we have the following corollary of Theorem 1.

Corollary 7 For every positive integer $n$,

$$
\begin{aligned}
& \left|g^{n+1}\left(a_{1}\right)\right|_{1 \# 2}=\left|g^{n+1}\left(a_{2} a_{4}\right)\right|_{1 \# 2}=\left|g^{n+1}\left(a_{3} a_{5}\right)\right|_{1 \# 2}=3^{n-1} \cdot\left(5 \cdot 3^{n+1}+2\right), \\
& \left|g^{n+1}\left(a_{1}\right)\right|_{2 \# 1}=\left|g^{n+1}\left(a_{2} a_{4}\right)\right|_{2 \# 1}=\left|g^{n+1}\left(a_{3} a_{5}\right)\right|_{2 \# 1}=3^{n-1} \cdot\left(5 \cdot 3^{n+1}-2\right) .
\end{aligned}
$$

3. Let $A$ be the three-letter ordered alphabet $A=\{a<b<c\}$. Define the morphism $h$ by

$$
\begin{aligned}
h: A^{*} & \rightarrow A^{*} \\
a & \mapsto a b a c a b c a c b a b c b a c b c \\
b & \mapsto a b a c a b c a c b c a b c b a b c \\
c & \mapsto a b a c a b c b a c b c a c b a b c
\end{aligned}
$$

This morphism was given to be square-free by Brandenburg in [6]. It fulfills the conditions of Theorem 1 with $p=3, A=A_{1} \cup A_{2} \cup A_{3}$ with $A_{1}=\{a\}, A_{2}=\{b\}, A_{3}=\{c\}$, and $m_{1}=m_{2}=m_{3}=6$, thus $M=18$. One has $|h(a)|_{1 \# 2}=70,|h(b)|_{1 \# 2}=|h(c)|_{1 \# 2}=66$, $|h(a)|_{2 \# 1}=38,|h(b)|_{2 \# 1}=|h(c)|_{2 \# 1}=42$.

Moreover, due to the particular form of the morphism $h$ (it is uniform, i.e., $|h(a)|=$ $\mid h\left(b|=| h(c) \mid\right.$, and for every $\left.x, y \in A,|h(x)|_{y}=6\right)$, one has $|h(x y)|_{1 \# 2}^{\text {ext }}=|h(x y)|_{2 \# 1}^{e x t}=108$ for every $x, y \in A$.

Then we have the following corollary of Theorem 1.

Corollary 8 For every $x \in A$ and for every positive integer $n$,

$$
\begin{aligned}
& \left|h^{n+1}(x)\right|_{1 \# 2}=6 \cdot 18^{n-1} \cdot\left(9 \cdot 18^{n+1}+40\right), \\
& \left|h^{n+1}(x)\right|_{2 \# 1}=6 \cdot 18^{n-1} \cdot\left(9 \cdot 18^{n+1}-40\right) .
\end{aligned}
$$

\section{Ordered Patterns with No Gaps and Morphisms}

In this last section we consider the problem of counting consecutive patterns in words generated by morphisms. Here the things are a little bit more complicated than in Section 3. 
Indeed the computation of external occurrences of such a pattern can be distorted by the fact that morphisms are allowed to be erasing. For example if $A=\left\{a_{1}<a_{2}<a_{3}\right\}$ and $f\left(a_{1}\right)=a_{2} a_{1}, f\left(a_{2}\right)=\varepsilon, f\left(a_{3}\right)=a_{3}$ then the word $f\left(a_{1} a_{2} a_{3}\right)$ contains an occurrence of the consecutive pattern 12 while $f\left(a_{1} a_{2}\right)$ and $f\left(a_{2} a_{3}\right)$ do not contain such an occurrence. Thus we have a priori to study more than only words of the form $f\left(a_{i} a_{j}\right)$ which were enough in the case of classical patterns. The result of this study is presented in Proposition 5 in which are given recurrence formulas for rises (occurrences of the ordered pattern 12), descents (occurrences of 21), and squares of one letter (occurrences of 11). We end again with some examples illustrating that our technique can provide exact formulas when the morphism is given.

\subsection{Rises, Descents, and Squares of $f^{n}$}

Let $k$ be an integer $(k \geq 2)$ and $A$ the $k$-letter ordered alphabet $A=\left\{a_{1}<a_{2}<\cdots<a_{k}\right\}$. Let $f$ be any morphism on $A$ : for $1 \leq i \leq k, f\left(a_{i}\right)=a_{i_{1}} \ldots a_{i_{p_{i}}}$ with $p_{i} \geq 0\left(p_{i}=0\right.$ if and only if $\left.f\left(a_{i}\right)=\varepsilon\right)$.

The vector of rises of $f^{n}$ is the $k$ vector whose $i$-th entry is the number of occurrences of the ordered pattern 12 in the word $f^{n}\left(a_{i}\right)$, i.e.,

$$
R\left(f^{n}\right)=\left(\left|f^{n}\left(a_{i}\right)\right|_{12}\right)_{1 \leq i \leq k}
$$

The vector of descents of $f^{n}$ is the $k$ vector whose $i$-th entry is the number of occurrences of the ordered pattern 21 in the word $f^{n}\left(a_{i}\right)$, i.e.,

$$
D\left(f^{n}\right)=\left(\left|f^{n}\left(a_{i}\right)\right|_{21}\right)_{1 \leq i \leq k}
$$

The vector of squares of one letter of $f^{n}$ is the $k$ vector whose $i$-th entry is the number of occurrences of the ordered pattern 11 in the word $f^{n}\left(a_{i}\right)$, i.e.,

$$
R_{2}\left(f^{n}\right)=\left(\left|f^{n}\left(a_{i}\right)\right|_{11}\right)_{1 \leq i \leq k} .
$$

Here again, as in Section 3, our goal is to obtain recurrence formulas giving the entries of $R\left(f^{n+1}\right), D\left(f^{n+1}\right)$, and $R_{2}\left(f^{n+1}\right)$.

We define two sequences of $k$ vectors, $\left(F\left(f^{n}\right)\right)_{n \in \mathbb{N}}$ and $\left(L\left(f^{n}\right)\right)_{n \in \mathbb{N}}$, where $F\left(f^{n}\right)[i]$ is the first letter of $f^{n}\left(a_{i}\right)$ and $L\left(f^{n}\right)[i]$ is the last letter of $f^{n}\left(a_{i}\right)$ if $f^{n}\left(a_{i}\right) \neq \varepsilon$, and $F\left(f^{n}\right)[i]=$ $L\left(f^{n}\right)[i]=0$ if $f^{n}\left(a_{i}\right)=\varepsilon$. Of course these two sequences take their values in a finite set: they are ultimately periodic. Thus they can be computed a priori from $f$.

Given a non-negative integer $n$, let $\aleph^{\prime}$ be the subset of $\aleph$ such that, for each $i \in \aleph$, $f^{n}\left(a_{i}\right) \neq \varepsilon$ if and only if $i \in \aleph^{\prime}$. We associate with the two vectors $F\left(f^{n}\right)$ and $L\left(f^{n}\right)$ an application $C_{n}^{12}: \aleph^{\prime} \times \aleph^{\prime} \rightarrow\{0,1\}$ defined by

$$
C_{n}^{12}(i, j)= \begin{cases}1, & \text { if } L\left(f^{n}\right)[i]<F\left(f^{n}\right)[j] \\ 0, & \text { if } L\left(f^{n}\right)[i] \geq F\left(f^{n}\right)[j] .\end{cases}
$$


Similarly we define

$$
C_{n}^{21}(i, j)= \begin{cases}1, & \text { if } L\left(f^{n}\right)[i]>F\left(f^{n}\right)[j] \\ 0, & \text { if } L\left(f^{n}\right)[i] \leq F\left(f^{n}\right)[j]\end{cases}
$$

and

$$
C_{n}^{11}(i, j)= \begin{cases}1, & \text { if } L\left(f^{n}\right)[i]=F\left(f^{n}\right)[j] \\ 0, & \text { if } L\left(f^{n}\right)[i] \neq F\left(f^{n}\right)[j]\end{cases}
$$

For any morphism $f$ on $A$, there exists a least integer $M_{f}\left(M_{f} \leq k\right.$ and $M_{f}$ depends only on $f$ ) such that, for every positive integer $n$ and every $a \in A, f^{n}(a)=\varepsilon$ if and only if $f^{M_{f}}(a)=\varepsilon$. By convention, if $f$ is a nonerasing morphism then $M_{f}=0$. The integer $M_{f}$ is known in the literature about $L$-systems as the mortality exponent of $f$ (see, e.g., [20]).

Now let $\ell$ be an integer, $1 \leq \ell \leq k$. One has $f\left(a_{\ell}\right)=a_{\ell_{1}} \ldots a_{\ell_{p_{\ell}}}$ and we denote by $\ell_{1}^{\prime} \ldots \ell_{p_{\ell}}^{\prime}$ the subsequence of $\ell_{1} \ldots \ell_{p_{\ell}}$ such that $f^{n+1}\left(a_{\ell}\right)=f^{n}\left(a_{\ell_{1}^{\prime}} \ldots a_{\ell_{p_{\ell}^{\prime}}}\right)$ for every $n \geq M_{f}$. This means that, for every $n \geq M_{f}$, a letter $a_{\ell_{i}}$ appears in $a_{\ell_{1}} \ldots a_{\ell_{p_{\ell}}}$ but not in $a_{\ell_{1}^{\prime}} \ldots a_{\ell_{p_{\ell}^{\prime}}^{\prime}}$ if and only if $f^{n}\left(a_{\ell_{i}}\right)=\varepsilon$. Of course $p_{\ell}^{\prime} \leq p_{\ell}$, and if $M_{f}=0$ then $p_{\ell}^{\prime}=p_{\ell}$ for each $1 \leq \ell \leq k$.

Here also, as in Section 3, the number of occurrences of the ordered pattern 12 in $f^{n+1}\left(a_{\ell}\right)=f^{n}\left(a_{\ell_{1}} \ldots a_{\ell_{\ell_{\ell}}}\right)=f^{n}\left(a_{\ell_{1}^{\prime}} \ldots a_{\ell_{p_{\ell}^{\prime}}}\right)\left(n \geq M_{f}\right)$ is obtained by adding two values:

- the number of occurrences of the ordered pattern 12 in each $f^{n}\left(a_{\ell_{i}}\right), 1 \leq i \leq p_{\ell}$. As in the previous case, this number is equal to $\sum_{t=1}^{k}\left|f^{n}\left(a_{t}\right)\right|_{12} \cdot m_{1, t, \ell}$,

- the number of external occurrences of the ordered pattern 12 in $f^{n}\left(a_{\ell_{i}^{\prime}} a_{\ell_{j}^{\prime}}\right)$ for each subsequence $a_{\ell_{i}^{\prime}} a_{\ell_{j}^{\prime}}$ of $f\left(a_{\ell}\right), 1 \leq i<j \leq p_{\ell}^{\prime}$. But the only possibility for 12 to be an external occurrence in $f^{n}\left(a_{\ell_{i}^{\prime}} a_{\ell_{j}^{\prime}}\right)$ is that $j=i+1$ and the last letter of $f^{n}\left(a_{\ell_{i}^{\prime}}\right)$ is smaller than the first letter of $f^{n}\left(a_{\ell_{j}^{\prime}}\right)$. Thus, the number of occurrences of such patterns is only the number of times $L\left(f^{n}\right)[i]<F\left(f^{n}\right)[i+1]$ with $i+1 \leq p_{\ell}^{\prime}$, i.e., the number of times $C_{n}^{12}\left(\ell_{i}^{\prime}, \ell_{i+1}^{\prime}\right)=1$ for $1 \leq i \leq p_{\ell}^{\prime}-1$.

We proceed similarly with the patterns 21 and 11 . Consequently we have the following proposition.

Proposition 5 For each letter $a_{\ell} \in A, f\left(a_{\ell}\right)=a_{\ell_{1}} \ldots a_{\ell_{p_{\ell}}}$, and for all $n \geq M_{f}$, let $\ell_{1}^{\prime} \ldots \ell_{p_{\ell}^{\prime}}^{\prime}$ be the subsequence of $\ell_{1} \ldots \ell_{p_{\ell}}$ such that $f^{n+1}\left(a_{\ell}\right)=f^{n}\left(a_{\ell_{1}^{\prime}} \ldots a_{\ell_{p_{\ell}^{\prime}}^{\prime}}\right)$ and $f^{n}\left(a_{\ell_{i}^{\prime}}\right) \neq \varepsilon, 1 \leq i \leq$ $p_{\ell}^{\prime}$. Then

$$
\left|f^{n+1}\left(a_{\ell}\right)\right|_{12}=\sum_{t=1}^{k}\left|f^{n}\left(a_{t}\right)\right|_{12} \cdot m_{1, t, \ell}+\sum_{i=1}^{p_{\ell}^{\prime}-1} C_{n}^{12}\left(\ell_{i}^{\prime}, \ell_{i+1}^{\prime}\right)
$$




$$
\begin{aligned}
& \left|f^{n+1}\left(a_{\ell}\right)\right|_{21}=\sum_{t=1}^{k}\left|f^{n}\left(a_{t}\right)\right|_{21} \cdot m_{1, t, \ell}+\sum_{i=1}^{p_{\ell}^{\prime}-1} C_{n}^{21}\left(\ell_{i}^{\prime}, \ell_{i+1}^{\prime}\right), \\
& \left|f^{n+1}\left(a_{\ell}\right)\right|_{11}=\sum_{t=1}^{k}\left|f^{n}\left(a_{t}\right)\right|_{11} \cdot m_{1, t, \ell}+\sum_{i=1}^{p_{\ell}^{\prime}-1} C_{n}^{11}\left(\ell_{i}^{\prime}, \ell_{i+1}^{\prime}\right) .
\end{aligned}
$$

\subsection{About the Repetitions of One Letter with No Gaps}

The case of $p$-repetitions of one letter is more complicated when no gaps are allowed. Indeed we have to find blocks of $p$ consecutive equal letters but, generally, this number $p$ is limited by a given value depending on the morphism itself. For example the Thue-Morse morphism $\mu$ (see Section 3.2) is such that $\mu^{n}\left(a_{1}\right)$ and $\mu^{n}\left(a_{2}\right)$ do not contain $a_{1} a_{1} a_{1}$ nor $a_{2} a_{2} a_{2}$ as factors, whatever be the value of $n$ ( $\mu$ generates cube-free words). This explains why in the previous section we only provide a formula giving the number of squares of one letter (the ordered pattern 11) in the words $f^{n}\left(a_{i}\right), 1 \leq i \leq k$. If we want to obtain a formula giving the number of $p$-powers of one letter (the ordered pattern $1^{p}$ ) for some $p \geq 3$ the computation of external repetitions (corresponding to $C_{n}^{11}$ in equation (8)) will become much more complicated.

\subsection{Some Examples}

We only give here a little number of examples illustrating Proposition 5 because the involved techniques are roughly the same as in the case of patterns with gaps. First we give three particular cases of families of morphisms in which the number of external occurrences of the ordered pattern is trivially obtained. Then we give examples of exact formulas in the well known cases of the Thue-Morse and the Fibonacci morphisms in order to compare with the results obtained in Section 3.2. We end by an example of a basic erasing morphism and one in which the value of the integer $M_{f}$ above is 2 .

\subsubsection{No External Rises}

Let us suppose that the morphism $f$ is such that, for all $i$ and $j, L(f)[i] \geq F(f)[j]$. According to equation (6), in this case, for each letter $a_{\ell} \in A, f\left(a_{\ell}\right)=a_{\ell_{1}} \ldots a_{\ell_{p_{\ell}}}$, and for all $n \geq M_{f}$,

$$
\left|f^{n+1}\left(a_{\ell}\right)\right|_{12}=\sum_{t=1}^{k}\left|f^{n}\left(a_{t}\right)\right|_{12} \cdot m_{1, t, \ell}
$$

Moreover, if the above inequality is strict then, according to equation (7),

$$
\left|f^{n+1}\left(a_{\ell}\right)\right|_{21}=\sum_{t=1}^{k}\left|f^{n}\left(a_{t}\right)\right|_{21} \cdot m_{1, t, \ell}+p_{\ell}^{\prime}-1 .
$$




\subsubsection{No External Descents}

If, conversely to the previous case, the morphism $f$ is such that, for all $i$ and $j, L(f)[i] \leq$ $F(f)[j]$ then, according to equation (7), for each letter $a_{\ell} \in A, f\left(a_{\ell}\right)=a_{\ell_{1}} \ldots a_{\ell_{p_{\ell}}}$, and for all $n \geq M_{f}$,

$$
\left|f^{n+1}\left(a_{\ell}\right)\right|_{21}=\sum_{t=1}^{k}\left|f^{n}\left(a_{t}\right)\right|_{21} \cdot m_{1, t, \ell}
$$

Moreover, if the above inequality is strict then, according to equation (6),

$$
\left|f^{n+1}\left(a_{\ell}\right)\right|_{12}=\sum_{t=1}^{k}\left|f^{n}\left(a_{t}\right)\right|_{12} \cdot m_{1, t, \ell}+p_{\ell}^{\prime}-1 .
$$

\subsubsection{No External Squares}

Now, if we suppose that the morphism $f$ is such that, for all $i$ and $j, L(f)[i] \neq F(f)[j]$ then, according to equation (8), for each letter $a_{\ell} \in A, f\left(a_{\ell}\right)=a_{\ell_{1}} \ldots a_{\ell_{p_{\ell}}}$, and for all $n \geq M_{f}$,

$$
\left|f^{n+1}\left(a_{\ell}\right)\right|_{11}=\sum_{t=1}^{k}\left|f^{n}\left(a_{t}\right)\right|_{11} \cdot m_{1, t, \ell}
$$

\subsubsection{The Thue-Morse Morphism}

For details on this morphism see Section 3.2. Here, $k=2$ and for all $1 \leq t, \ell \leq 2, m_{1, t, \ell}=1$. Since $\mu$ is nonerasing, $M_{\mu}=0$. Moreover for any integer $n \geq 0$,

$$
\begin{gathered}
C_{n}^{12}(1,2)=C_{n}^{21}(2,1)= \begin{cases}1, & \text { if } n \text { is even } \\
0, & \text { if } n \text { is odd }\end{cases} \\
C_{n}^{12}(2,1)=C_{n}^{21}(1,2)=0 \text { and } C_{n}^{11}(1,2)=C_{n}^{11}(2,1)= \begin{cases}1, & \text { if } n \text { is odd } \\
0, & \text { if } n \text { is even. }\end{cases}
\end{gathered}
$$

Thus, from equations (6), (7), and (8) we obtain, for every $n \geq 0$,

$$
\begin{aligned}
& \left|\mu^{n+1}\left(a_{1}\right)\right|_{12}=\left|\mu^{n}\left(a_{1}\right)\right|_{12}+\left|\mu^{n}\left(a_{2}\right)\right|_{12}+ \begin{cases}1, & \text { if } n \text { is even } \\
0, & \text { if } n \text { is odd }\end{cases} \\
& \left|\mu^{n+1}\left(a_{2}\right)\right|_{12}=\left|\mu^{n}\left(a_{1}\right)\right|_{12}+\left|\mu^{n}\left(a_{2}\right)\right|_{12} \\
& \left|\mu^{n+1}\left(a_{1}\right)\right|_{21}=\left|\mu^{n}\left(a_{1}\right)\right|_{21}+\left|\mu^{n}\left(a_{2}\right)\right|_{21} \\
& \left|\mu^{n+1}\left(a_{2}\right)\right|_{21}=\left|\mu^{n}\left(a_{1}\right)\right|_{21}+\left|\mu^{n}\left(a_{2}\right)\right|_{21}+ \begin{cases}1, & \text { if } n \text { is even } \\
0, & \text { if } n \text { is odd }\end{cases} \\
& \left|\mu^{n+1}\left(a_{1}\right)\right|_{11}=\left|\mu^{n+1}\left(a_{2}\right)\right|_{11}=2 \cdot\left|\mu^{n}\left(a_{1}\right)\right|_{11}+ \begin{cases}1, & \text { if } n \text { is odd } \\
0, & \text { if } n \text { is even. }\end{cases}
\end{aligned}
$$


Since $R(\mu)=\left[\begin{array}{ll}1 & 0\end{array}\right], D(\mu)=\left[\begin{array}{ll}0 & 1\end{array}\right]$ and $R_{2}(\mu)=\left[\begin{array}{ll}0 & 0\end{array}\right]$ we obtain again a rather trivial result.

Corollary 9 For any integer $n \geq 0$,

$$
\begin{aligned}
& R\left(\mu^{2 n}\right)=\left[\begin{array}{ll}
\frac{4^{n}-1}{3} & \frac{4^{n}-1}{3}
\end{array}\right]=D\left(\mu^{2 n}\right)=R_{2}\left(\mu^{2 n}\right) \\
& R\left(\mu^{2 n+1}\right)=\left[\begin{array}{ll}
\frac{2\left(4^{n}-1\right)}{3}+1 & \frac{2\left(4^{n}-1\right)}{3}
\end{array}\right] \\
& D\left(\mu^{2 n+1}\right)=\left[\begin{array}{ll}
\frac{2\left(4^{n}-1\right)}{3} & \frac{2\left(4^{n}-1\right)}{3}+1
\end{array}\right] \\
& R_{2}\left(\mu^{2 n+1}\right)=\left[\begin{array}{ll}
\frac{2\left(4^{n}-1\right)}{3} & \frac{2\left(4^{n}-1\right)}{3}
\end{array}\right] .
\end{aligned}
$$

\subsubsection{The Fibonacci Morphism}

For details on this morphism see Section 3.2. Here again $k=2$ and $M_{\varphi}=0$. First we note that for $n \geq 1$, since $\varphi\left(a_{2}\right)=a_{1}$, one has $\left|\varphi^{n}\left(a_{2}\right)\right|_{x y}=\left|\varphi^{n-1}\left(a_{1}\right)\right|_{x y}$ for $x y=12, x y=21$, and $x y=11$. Moreover we are in case 6.3 .1 above thus $\left|\varphi^{n+1}\left(a_{1}\right)\right|_{12}=\left|\varphi^{n}\left(a_{1}\right)\right|_{12} \cdot m_{1,1,1}+$ $\left|\varphi^{n}\left(a_{2}\right)\right|_{12} \cdot m_{1,2,1}$ for every $n \geq 1$.

Now $m_{1,1,1}=m_{1,2,1}=1$ and, for any positive integer $n, C_{n}^{21}(1,2)= \begin{cases}1, & \text { if } n \text { is odd } \\ 0, & \text { if } n \text { is even }\end{cases}$ and $C_{n}^{11}(1,2)=\left\{\begin{array}{ll}1, & \text { if } n \text { is even } \\ 0, & \text { if } n \text { is odd. }\end{array}\right.$ Thus, from equations $(7)$ and (8) we obtain, for every $n \geq 1$

$$
\begin{aligned}
& \left|\varphi^{n+1}\left(a_{1}\right)\right|_{21}=\left|\varphi^{n}\left(a_{1}\right)\right|_{21}+\left|\varphi^{n}\left(a_{2}\right)\right|_{21}+ \begin{cases}1, & \text { if } n \text { is odd } \\
0, & \text { if } n \text { is even }\end{cases} \\
& \left|\varphi^{n+1}\left(a_{1}\right)\right|_{11}=\left|\varphi^{n}\left(a_{1}\right)\right|_{11}+\left|\varphi^{n}\left(a_{2}\right)\right|_{11}+ \begin{cases}1, & \text { if } n \text { is even } \\
0, & \text { if } n \text { is odd }\end{cases}
\end{aligned}
$$

Since $R(\varphi)=\left[\begin{array}{ll}1 & 0\end{array}\right]$ and $D(\varphi)=R_{2}(\varphi)=\left[\begin{array}{ll}0 & 0\end{array}\right]$ we have again a well known result.

Corollary 10 For any integer $n \geq 1$,

$$
\begin{aligned}
R\left(\varphi^{n}\right) & =\left[\begin{array}{ll}
F_{n-1} & F_{n-2}
\end{array}\right] \\
D\left(\varphi^{2 n}\right) & =\left[\begin{array}{ll}
F_{2 n-1} & F_{2 n-2}-1
\end{array}\right]=R_{2}\left(\varphi^{2 n+1}\right) \\
R_{2}\left(\varphi^{2 n}\right) & =\left[\begin{array}{ll}
F_{2 n-2}-1 & F_{2 n-3}
\end{array}\right]=D\left(\varphi^{2 n-1}\right) .
\end{aligned}
$$




\subsubsection{Erasing Morphisms}

Let $A$ be the four-letter ordered alphabet $A=\left\{a_{1}<a_{2}<a_{3}<a_{4}\right\}$.

1. Here we consider the erasing morphism $f$, given in Section 5.5, defined on $A$ by

$$
\begin{aligned}
f\left(a_{1}\right) & =a_{1} a_{3} a_{2} a_{4} \\
f\left(a_{2}\right) & =\varepsilon \\
f\left(a_{3}\right) & =a_{1} a_{4} \\
f\left(a_{4}\right) & =a_{2} a_{3} .
\end{aligned}
$$

We have $M_{f}=1$.

First, we remark that, for any positive integer $n, f^{n}\left(a_{2}\right)=\varepsilon$ and thus $\left|f^{n}\left(a_{2}\right)\right|_{x y}=0$ for $x y=12, x y=21$, and $x y=11$. Here again we are in case 6.3 .1 above and thus, for $n \geq 1$,

$$
\begin{aligned}
\left|f^{n+1}\left(a_{1}\right)\right|_{12} & =\left|f^{n}\left(a_{1}\right)\right|_{12}+\left|f^{n}\left(a_{2}\right)\right|_{12}+\left|f^{n}\left(a_{3}\right)\right|_{12}+\left|f^{n}\left(a_{4}\right)\right|_{12} \\
& =\left|f^{n}\left(a_{1}\right)\right|_{12}+\left|f^{n}\left(a_{3}\right)\right|_{12}+\left|f^{n}\left(a_{4}\right)\right|_{12} \\
\left|f^{n+1}\left(a_{3}\right)\right|_{12} & =\left|f^{n}\left(a_{1}\right)\right|_{12}+\left|f^{n}\left(a_{4}\right)\right|_{12} \\
\left|f^{n+1}\left(a_{4}\right)\right|_{12} & =\left|f^{n}\left(a_{3}\right)\right|_{12} .
\end{aligned}
$$

Moreover, the values of the number $p_{\ell}^{\prime}$ of Proposition 5 are $p_{1}^{\prime}=3, p_{3}^{\prime}=2, p_{4}^{\prime}=1$ and, since the inequality $L(f)[i] \geq F(f)[j]$ is strict for all the values of $i, j$, one has for every $n \geq 1$

$$
\begin{aligned}
\left|f^{n+1}\left(a_{1}\right)\right|_{21} & =\left|f^{n}\left(a_{1}\right)\right|_{21}+\left|f^{n}\left(a_{3}\right)\right|_{21}+\left|f^{n}\left(a_{4}\right)\right|_{21}+p_{1}^{\prime}-1 \\
& =\left|f^{n}\left(a_{1}\right)\right|_{21}+\left|f^{n}\left(a_{3}\right)\right|_{21}+\left|f^{n}\left(a_{4}\right)\right|_{21}+2 \\
\left|f^{n+1}\left(a_{3}\right)\right|_{21} & =\left|f^{n}\left(a_{1}\right)\right|_{21}+\left|f^{n}\left(a_{4}\right)\right|_{21}+1 \\
\left|f^{n+1}\left(a_{4}\right)\right|_{21} & =\left|f^{n}\left(a_{3}\right)\right|_{21} .
\end{aligned}
$$

But $\left|f\left(a_{1}\right)\right|_{12}=2,\left|f\left(a_{3}\right)\right|_{12}=1,\left|f\left(a_{4}\right)\right|_{12}=1$ and $\left|f\left(a_{1}\right)\right|_{21}=1,\left|f\left(a_{3}\right)\right|_{21}=0,\left|f\left(a_{4}\right)\right|_{21}=$ 0 . Thus, we deduce easily that $\left|f^{n}\left(a_{i}\right)\right|_{21}=\left|f^{n}\left(a_{i}\right)\right|_{12}-1$ for $i=1, i=3$, and $i=4$.

To end, we are also in case 6.3 .3 above. Consequently, for every $n \geq 1$,

$$
\begin{aligned}
\left|f^{n+1}\left(a_{1}\right)\right|_{11} & =\left|f^{n}\left(a_{1}\right)\right|_{11}+\left|f^{n}\left(a_{3}\right)\right|_{11}+\left|f^{n}\left(a_{4}\right)\right|_{11} \\
\left|f^{n+1}\left(a_{3}\right)\right|_{11} & =\left|f^{n}\left(a_{1}\right)\right|_{11}+\left|f^{n}\left(a_{4}\right)\right|_{11} \\
\left|f^{n+1}\left(a_{4}\right)\right|_{11} & =\left|f^{n}\left(a_{3}\right)\right|_{11} .
\end{aligned}
$$

But, since $\left|f\left(a_{1}\right)\right|_{11}=\left|f\left(a_{3}\right)\right|_{11}=\left|f\left(a_{4}\right)\right|_{11}=0$, this implies that $\left|f^{n}\left(a_{1}\right)\right|_{11}=\left|f^{n}\left(a_{3}\right)\right|_{11}=$ $\left|f^{n}\left(a_{4}\right)\right|_{11}=0$ for any positive integer $n$. So it remains to calculate the values of $\left|f^{n+1}\left(a_{1}\right)\right|_{12}$ and $\left|f^{n+1}\left(a_{3}\right)\right|_{12}$. 
Since $f\left(a_{1}\right)=a_{1} a_{3} a_{2} a_{4}$ and $f\left(a_{3} a_{4}\right)=a_{1} a_{4} a_{2} a_{3}$, it is clear that $\left|f^{n}\left(a_{1}\right)\right|_{a_{i}}=2^{n-1}$ for each $1 \leq i \leq 4$ and $n \geq 1$. Thus, since there are no external occurrences of the ordered pattern 12 in a word $f^{n}\left(a_{i}\right)$ regardless of the value of $n$, one has for every $n \geq 1$

$$
\begin{aligned}
\left|f^{n+1}\left(a_{1}\right)\right|_{12} & =2^{n-1} \cdot\left(\left|f\left(a_{1}\right)\right|_{12}+\left|f\left(a_{3}\right)\right|_{12}+\left|f\left(a_{4}\right)\right|_{12}\right) \\
& =2^{n-1} \cdot 4 \\
& =2^{n+1} .
\end{aligned}
$$

In the same manner, $\left|f^{n}\left(a_{3}\right)\right|_{a_{1}}=\left|f^{n}\left(a_{3}\right)\right|_{a_{4}}=\left\{\begin{array}{ll}\frac{2^{n}-1}{n^{3}}, & \text { if } n \text { is even } \\ \frac{2^{n^{3}}}{3}, & \text { if } n \text { is odd }\end{array}\right.$ for every positive

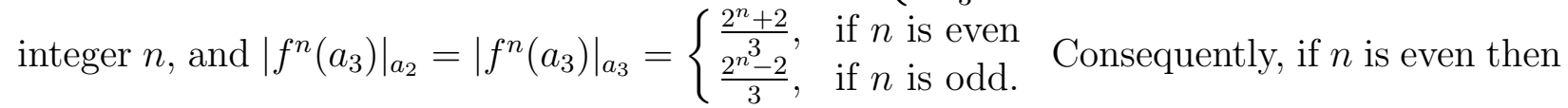

$$
\begin{aligned}
\left|f^{n+1}\left(a_{3}\right)\right|_{12} & =\frac{2^{n}-1}{3} \cdot\left(\left|f\left(a_{1}\right)\right|_{12}+\left|f\left(a_{4}\right)\right|_{12}\right)+\frac{2^{n}+2}{3} \cdot\left|f\left(a_{3}\right)\right|_{12} \\
& =\frac{3\left(2^{n}-1\right)}{3}+\frac{2^{n}+2}{3} \\
& =\frac{2^{n+2}-1}{3}
\end{aligned}
$$

and if $n$ is odd then $\left|f^{n+1}\left(a_{3}\right)\right|_{12}=\frac{3\left(2^{n}+1\right)}{3}+\frac{2^{n}-2}{3}=\frac{2^{n+2}+1}{3}$.

Starting from $R(f)=\left[\begin{array}{llll}2 & 0 & 1 & 1\end{array}\right]$, the above results are summarized in the following result.

Corollary 11 For any integer $n \geq 1, R_{2}\left(f^{n}\right)=\left[\begin{array}{llll}0 & 0 & 0 & 0\end{array}\right]$ and

$$
\begin{aligned}
& \text { if } n \text { is even }\left\{\begin{array}{l}
R\left(f^{n}\right)=\left[\begin{array}{llll}
2^{n} & 0 & \frac{2^{n+1}+1}{3} & \frac{2^{n}-1}{3}
\end{array}\right] \\
D\left(f^{n}\right)=\left[\begin{array}{llll}
2^{n}-1 & 0 & \frac{2^{n+1}-2}{3} & \frac{2^{n}-4}{3}
\end{array}\right],
\end{array}\right. \\
& \text { if } n \text { is odd }\left\{\begin{array}{l}
R\left(f^{n}\right)=\left[\begin{array}{llll}
2^{n} & 0 & \frac{2^{n+1}-1}{3} & \frac{2^{n}+1}{3}
\end{array}\right] \\
D\left(f^{n}\right)=\left[\begin{array}{llll}
2^{n}-1 & 0 & \frac{2^{n+1}-4}{3} & \frac{2^{n}-2}{3}
\end{array}\right] .
\end{array}\right.
\end{aligned}
$$

2. Now we consider the erasing morphism $g$ defined on $A$ by

$$
\begin{aligned}
& g\left(a_{1}\right)=a_{1} a_{2} a_{4} a_{3} \\
& f\left(a_{2}\right)=a_{3} \\
& f\left(a_{3}\right)=\varepsilon \\
& f\left(a_{4}\right)=a_{1} a_{2} a_{4} .
\end{aligned}
$$

Here we have $M_{f}=2$, i.e., we must be careful of the fact that $g^{n}\left(a_{2}\right)=\varepsilon$ only for $n=2$. Thus the recurrence formulas giving the values for $g^{n+1}$ from those for $g^{n}$ must be given for $n \geq 2$, which means that the particular cases are those for both $g$ and $g^{2}$.

Let $n \geq 2$. Since $g^{n}\left(a_{2}\right)=g^{n}\left(a_{3}\right)=\varepsilon$ one has, for $x y=12, x y=21$, and $x y=11$, $\left|g^{n+1}\left(a_{2}\right)\right|_{x y}=\left|g^{n+1}\left(a_{3}\right)\right|_{x y}=0$ and $\left|g^{n+1}\left(a_{1}\right)\right|_{x y}=\left|g^{n+1}\left(a_{4}\right)\right|_{x y}$. We are in case 6.3.1, so 
$\left|g^{n+1}\left(a_{1}\right)\right|_{12}=2 \cdot\left|g^{n}\left(a_{1}\right)\right|_{12}$. Now, $C_{n}^{21}(1,4)=1$, hence $\left|g^{n+1}\left(a_{1}\right)\right|_{21}=2 \cdot\left|g^{n}\left(a_{1}\right)\right|_{21}+1$. To end, $C_{n}^{11}(1,4)=0$ and thus $\left|g^{n+1}\left(a_{1}\right)\right|_{11}=2 \cdot\left|g^{n}\left(a_{1}\right)\right|_{11}$.

Consequently, we obtain the following result.

Corollary $12 R(g)=\left[\begin{array}{llll}2 & 0 & 0 & 2\end{array}\right], D(g)=\left[\begin{array}{llll}1 & 0 & 0 & 0\end{array}\right], R_{2}(g)=\left[\begin{array}{llll}0 & 0 & 0 & 0\end{array}\right]$, and, for any integer $n \geq 2$,

$$
\begin{aligned}
R\left(g^{n}\right) & =\left[\begin{array}{llll}
2^{n} & 0 & 0 & 2^{n}
\end{array}\right] \\
D\left(g^{n}\right) & =\left[\begin{array}{lllll}
2^{n-1}+2^{n-2}-1 & 0 & 0 & 2^{n-1}+2^{n-2}-1
\end{array}\right] \\
R_{2}\left(g^{n}\right) & =\left[\begin{array}{llll}
2^{n-2} & 0 & 0 & 2^{n-2}
\end{array}\right] .
\end{aligned}
$$

\section{Acknowledgements}

The authors are grateful to the anonymous referee for numerous valuable comments and suggestions.

\section{References}

[1] J.-P. Allouche, J. Shallit, Sums of digits, overlaps, and palindromes, Discrete Math. \& Theoret. Comput. Sci. 4 (2000), 1-10.

[2] J.-P. Allouche, J. Shallit, Automatic sequences: theory, applications, generalizations, Cambridge University Press, Cambridge, UK, 2003.

[3] S. Arshon, Démonstration de l'existence de suites asymétriques infinies, Mat. Sb. 44 (1937), 769-777 (in Russian), 777-779 (French summary).

[4] E. Babson, E. Steingrímsson, Generalized permutation patterns and a classification of the Mahonian statistics, Sém. Lothar. Comb. 44 (2000), Art. B44b, 18 pp.

[5] J. Berstel, Mots sans carré et morphismes itérés, Discrete Math. 29 (1980), 235-244.

[6] F.-J. Brandenburg, Uniformly growing $k$-th power-free homomorphisms, Theoret. Comput. Sci. 23 (1983), 69-82.

[7] A. Burstein, Enumeration of words with forbidden patterns, Ph.D. thesis, University of Pennsylvania, 1998.

[8] A. Burstein, T. Mansour, Words restricted bypatterns with at most 2 distinct letters, Elect. J. Combin. 9:2 (2002), \#R3.

[9] A. Burstein, T. Mansour, Words restricted by 3-letter generalized multipermutation patterns, Annals of Combin. 7 (2003), 1-14.

[10] A. Burstein, T. Mansour, Counting occurrences of some subword patterns, Discrete Math. \& Theoret. Comput. Sci. 6:1 (2003), 1-12. 
[11] A. Clatesson, Generalised Pattern Avoidance, European J. Combin. 22 no. 7 (2001), 961-971.

[12] A. Coвнam, Uniform tag sequences, Math. Systems Th. 6 (1972), 164-192.

[13] J. CuRRIE, No iterated morphism generates any Arshon sequence of odd order, Discrete Math. 259 (2002), 277-283.

[14] S. Fossé, G. Richomme, Some characterizations of Parikh matrix equivalent binary words, Inform. Proc. Letters 92 (2004), 77-82.

[15] S. Istrail, On irreducible languages and nonrational numbers, Bull. Math. Soc. Sci. Math. R. S. Roumanie 21 (1977), 301-308.

[16] S. Kitaev, There are no iterated morphisms that define the Arshon sequence and the sigma-sequence, J. of Automata, Languages and Combin. 8 (2003), 43-50.

[17] S. Kitaev, Multi-avoidance of generalised patterns, Discrete Math. 260 (2003), 89-100.

[18] S. Kitaev, T. Mansour, P. SÉÉBold, The Peano curve and counting occurrences of some patterns, J. of Automata, Languages and Combin. 9 (2004) 4, 439-455.

[19] D. E. Knuth, The Art of Computer Programming, 2nd ed., Addison-Wesley, Reading, MA, 1973.

[20] F. Levé, G. Richomme, On a conjecture about finite fixed points of morphisms, Theoret. Comput. Sci. 339-1 (2005), 103-128.

[21] M. Lothaire, Combinatorics on Words, vol. 17 of Encyclopedia of Mathematics and Applications, Addison-Wesley, Reading, Mass., 1983.

Reprinted in the Cambridge Mathematical Library, Cambridge University Press, Cambridge, UK, 1997.

[22] M. Lothaire, Algebraic Combinatorics on Words, vol.90 of Encyclopedia of Mathematics and its Applications, Cambridge University Press, Cambridge, UK, 2002.

[23] A. Mateescu, A. Salomaa, K. Salomaa, S. Yu, A sharpening of the Parikh mapping, Theoret. Informatics Appl. 35 (2001), 551-564.

[24] M. Morse, Recurrent geodesics on a surface of negative curvature, Trans. Amer. Math. Soc. 22 (1921), $84-100$.

[25] M. E. Prouhet, Mémoire sur quelques relations entre les puissances des nombres, Comptes Rendus Acad. Sci. Paris 33 (1851), 225.

[26] G. Rozenberg, A. Salomaa (Eds), Handbook of Formal Languages Vol. 1, Springer, 1997.

[27] A. SalomaA, Jewels of Formal Language Theory, Computer Science Press, 1981.

[28] P. SÉÉBold, About some overlap-free morphisms on a $n$-letter alphabet, J. of Automata, Languages and Combin. 7 (2002), 579-597.

[29] P. SÉÉBold, On some generalizations of the Thue-Morse morphism, Theoret. Comput. Sci. 292-1 (2003), 283-298.

[30] R. Simion, F. Schmidt, Restricted permutations, European J. Combin. 6 no. 4 (1985), 383-406.

[31] A. Thue, Über die gegenseitige Lage gleicher Teile gewisser Zeichenreihen, Vidensk.-Selsk. Skrifter. I. Mat. Nat. Kl. 1 Kristiania (1912), 1-67.

Reprinted in Selected Mathematical Papers of Axel Thue, T. Nagell, editor, Universitetsforlaget, Oslo, 1977, 413-478. 